
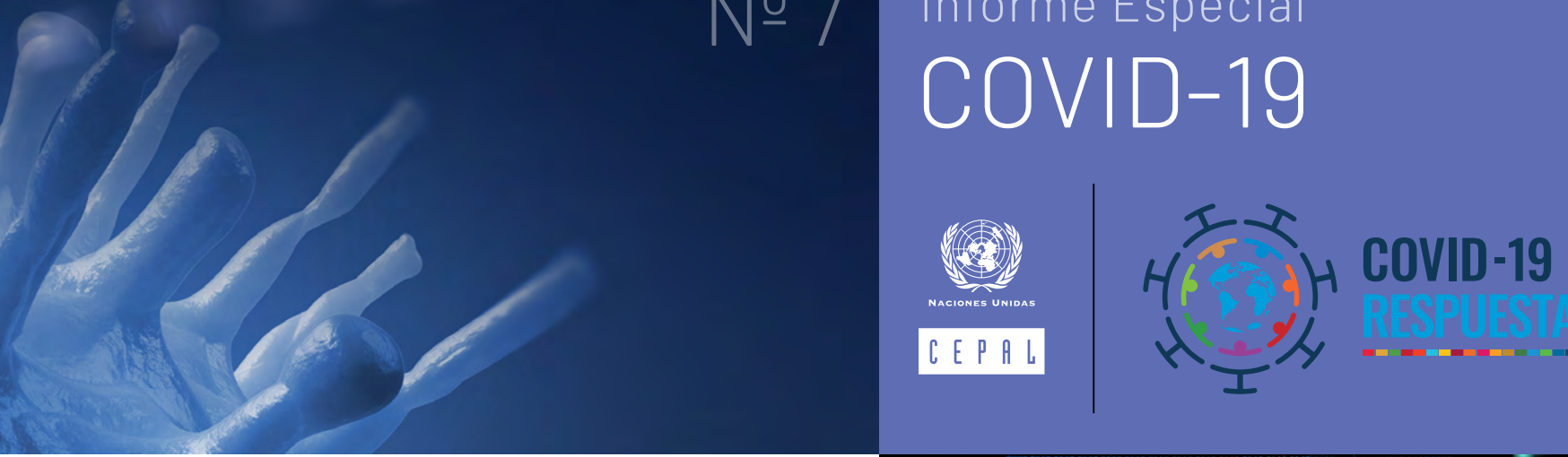

\title{
Universalizar el acceso a las tecnologías digitales para enfrentar los efectos del COVID-19
}

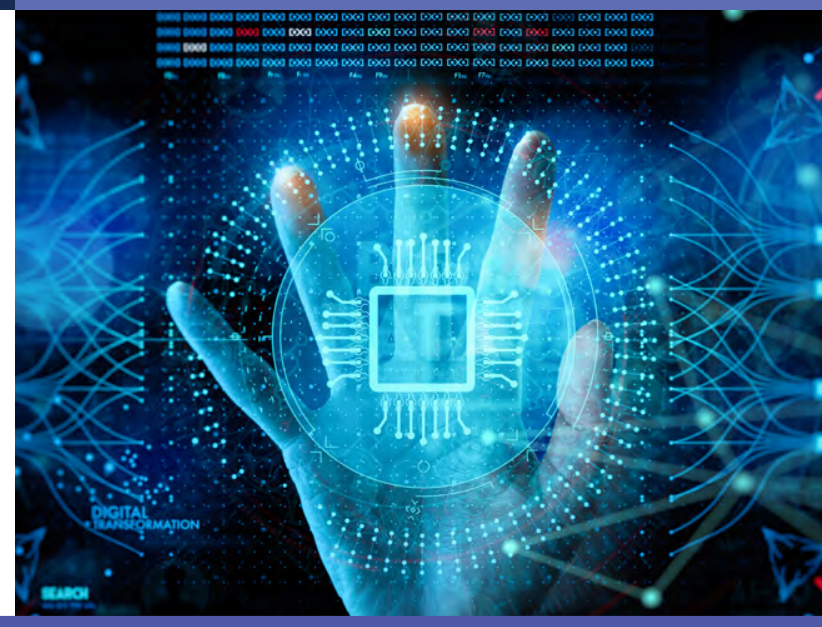

\section{A. Avance y limitaciones de la digitalización en la pandemia ${ }^{1}$}

- Las tecnologías digitales han sido esenciales para el funcionamiento de la economía y la sociedad durante la crisis de la pandemia de la enfermedad por coronavirus (COVID-19). Las redes y la infraestructura de comunicaciones se utilizan de manera cada vez más intensiva para actividades productivas, educacionales, de la salud, y de relacionamiento y entretenimiento. Avances que se preveía que demorarían años en concretarse, se han producido en pocos meses.

- Las soluciones digitales en las áreas de la salud, la educación, el comercio y el trabajo tienen un papel preponderante en la lucha contra el COVID-19 ya que facilitan el distanciamiento físico y viabilizan cierto funcionamiento del sistema socioeconómico.

- Los datos de movilidad durante los primeros meses de las cuarentenas muestran un mundo paralizado en lo físico, pero no en lo virtual.

- Los datos de desplazamientos de las personas en el inicio de la cuarentena muestran que, en los países de la región, la concurrencia a locales de venta de alimentos y farmacias disminuyó un $\mathbf{5 1 \% \text { , }}$ a establecimientos de comercio de productos no esenciales $y$ esparcimiento, cerca de un $\mathbf{7 5 \%}$, y a lugares de trabajo, alrededor de un $\mathbf{4 5 \%}$. Gran parte de la población mantiene medidas de aislamiento como forma de prevención obligatoria o voluntaria.

- Al mismo tiempo, el tráfico en sitios web y el uso de aplicaciones de teletrabajo, educación en línea y compras en línea revelan un significativo aumento del uso de soluciones digitales. Entre el primer y segundo trimestre de 2020, el uso de soluciones de teletrabajo aumentó un $324 \%$ y la educación en línea, más del $\mathbf{6 0} \%$ (véase el gráfico 1).

\section{Índice}

A. Avance y limitaciones de la digitalización en la pandemia

1. La brecha de acceso profundiza las desigualdades

2. El bajo ingreso de los hogares limita el acceso a Internet y las posibilidades de uso de aplicaciones móviles...

3. EI COVID-19 como acelerador de la discusión sobre la protección de los datos y la privacidad.

4. Regulación y defensa de la competencia..

B. Medidas de política y soluciones digitales

C. Hacia la reactivación.

1. La pospandemia: nueva realidad y cambio estructural.

2. Cinco líneas de acción. 
Gráfico 1 | América Latina (5 países)a: cambios en el nivel de actividad según sector entre el primer y segundo trimestre de 2020 en función del tráfico en sitios web y uso de aplicaciones

(En porcentajes)

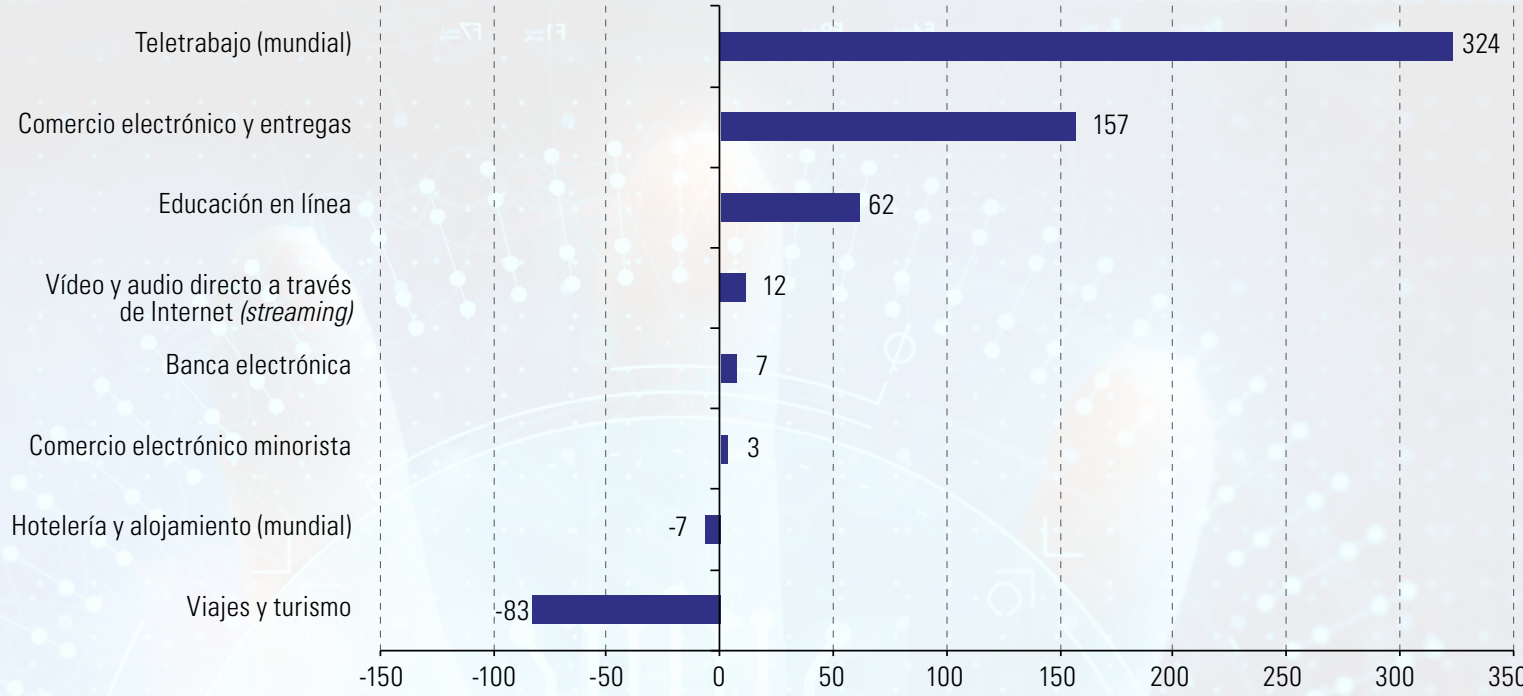

Fuente: Comisión Económica para América Latina y el Caribe (CEPAL), sobre la base de datos de SimilarWeb [en línea] similarweb.com [fecha de consulta: 3 de agosto de 2020]

Nota: Las categorías hotelería y alojamiento y teletrabajo incluyen datos globales, dado que los sitios no pueden asociarse con usuarios de un determinado país. Las aplicaciones de teletrabajo incluyen: zoom.us, meet.google.com, teams.microsoft.com, webex.com, y slack.com.; las de comercio electrónico y entregas: rappi.com, pedidosya.com, glovoapp.com, cornershop.com, ubereats.com.; las de educación en línea: uba.ar, unc.edu.ar, mineduc.cl, inacap.cl, uc.cl, uchile.cl, senasofiaplus.edu.co, brainly.lat, www.unad.edu.co, unal.edu.co, unam.mx, blackboard.com, edmodo.com, brainly.com.br, brasilescola.uol.com.br, colaboraread.com.br, qconcursos.com.; las de vídeo y audio directo a través de Internet (streaming): youtube.com, netflix.com; las de banca electrónica: itau.com.br, banco.bradesco, santander.com.br, bancochile.cl, santander.cl, santander.com.ar, banamex.com, bbva.mx.; las de comercio electrónico minorista: mercadolibre.com.mx, amazon.com.mx, mercadolibre.cl, falabella.cl, olx.com.co, mercadolibre.com.co, mercadolivre.com.br, olx.com.br, mercadolibre.com.ar, cotodigital3.com.ar.; las de hotelería y alojamiento: booking.com, agoda.com, airbnb.com, hotels.com, trivago.com, y las de viajes y turismo: airbnb.mx, volaris.com, booking.com.co, avianca.com.co, latam.com, booking.cl, booking.com.ar, despegar.com.ar, booking.com.br, latam.com/pt-br/

a La información empleada corresponde a: Argentina, Brasil, Chile, Colombia y México.

- El desarrollo y la adopción de soluciones tecnológicas están condicionados por factores estructurales: una heterogénea estructura productiva, un mercado laboral con una marcada informalidad y precariedad, una clase media vulnerable, un debilitado Estado de bienestar, una infraestructura digital deficiente y restricciones socioeconómicas al acceso y la conectividad.

- Los países de América Latina y el Caribe han adoptado medidas para impulsar el uso de esas soluciones tecnológicas y cautelar la continuidad de los servicios de telecomunicaciones. EI alcance de esas acciones es limitado por las brechas en el acceso y uso de esas tecnologías y las velocidades de conexión.

\section{La brecha de acceso profundiza las desigualdades}

- La conectividad, entendida como el servicio de banda ancha con una velocidad adecuada y la tenencia de dispositivos de acceso, condiciona el derecho a la salud, la educación y el trabajo, al tiempo que puede aumentar las desigualdades socioeconómicas.

- La conectividad es una condición necesaria, aunque no suficiente, para apropiarse del valor que generan las tecnologías digitales.

- En 2019, el 66,7\% de los habitantes de la región tenían conexión a Internet. El tercio restante tiene un acceso limitado o no tiene acceso a las tecnologías digitales debido a su condición económica y social, en particular su edad y localización.

- En 12 países de la región, la cifra de los hogares del quintil de ingresos más alto (quintil V) que tiene conexión a Internet es un $81 \%$, en promedio; las cifras correspondientes a los hogares del primer y segundo quintil es del $38 \%$ y el $53 \%$ respectivamente (véase el gráfico 2). En países como el Brasil y Chile, más del $60 \%$ de los hogares de los hogares del primer quintil tiene conexión a Internet, mientras que en Bolivia (Estado Plurinacional de), el Paraguay y el Perú, solo el 3\% la tiene. Ese bajo porcentaje limita o impide el acceso al teletrabajo, la educación en 
línea y los servicios de salud electrónica, así como a otros bienes y servicios ofrecidos por las plataformas e instituciones públicas, lo que amplía las brechas preexistentes.

• En 2018, casi 23 millones de los hogares - la mitad de los hogares sin conexión a Internetse ubicaban en los dos quintiles más bajos de la distribución del ingreso (quintiles I y II).

Gráfico 2 | América Latina (12 países): hogares con y sin conexión a Internet, por quintil de ingresos, 2018 (En porcentajes)

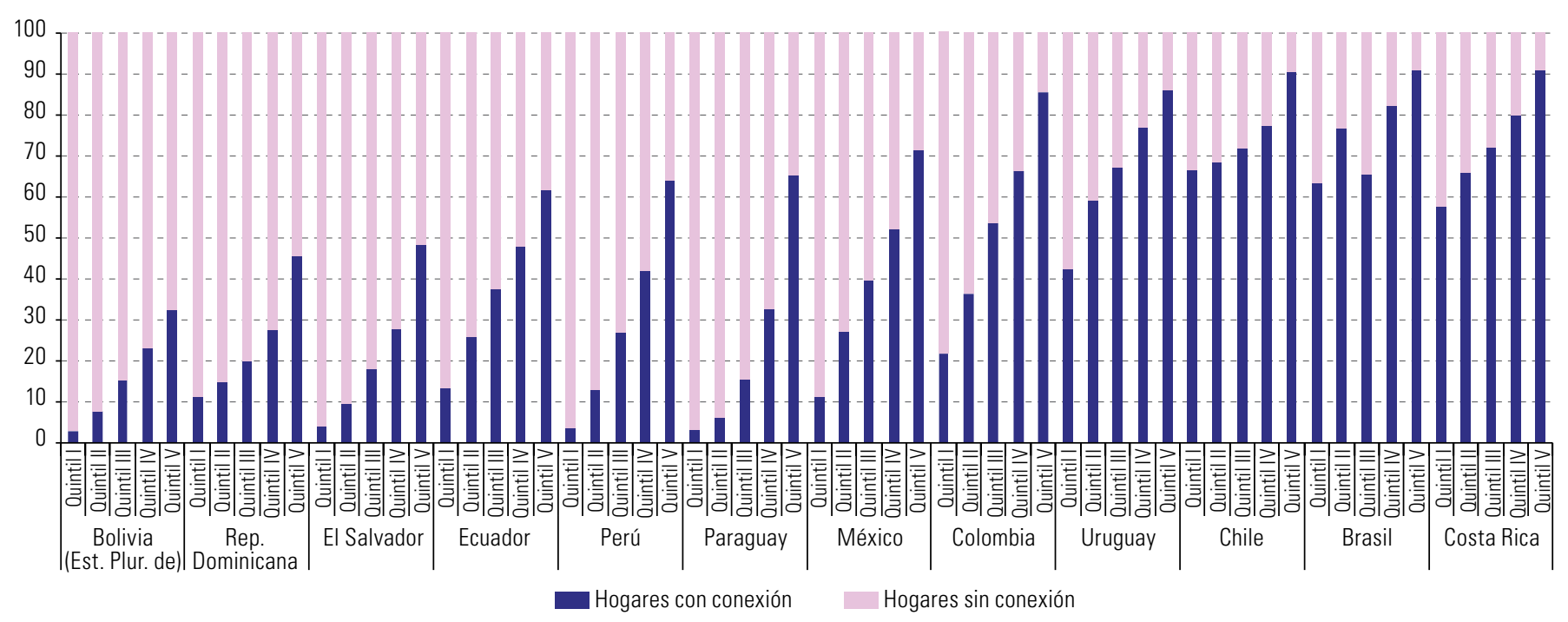

Fuente: Comisión Económica para América Latina y el Caribe (CEPAL), Observatorio Regional de Banda Ancha (ORBA), sobre la base de información del Banco de Datos de Encuestas de Hogares (BADEHOG).

Nota: Los datos estadísticos correspondientes al Brasil, Chile, Costa Rica, el Ecuador, El Salvador, el Paraguay y el Uruguay incluyen Internet móvil. Los datos de todos los países corresponden a 2018; los datos correspondientes a Chile y el Ecuador corresponden a 2017.

- Las diferencias en la conectividad entre la zona urbana y la rural son significativas. En la región, el $67 \%$ de los hogares urbanos está conectado a Internet, en tanto que en las zonas rurales solo lo está el 23\% de ellos. En algunos países, como Bolivia (Estado Plurinacional de), El Salvador, el Paraguay y el Perú, más del $\mathbf{9 0 \%}$ de los hogares rurales no cuentan con conexión a Internet. Incluso en países en mejor situación, como Chile, Costa Rica y el Uruguay, solo cerca de la mitad de los hogares rurales están conectados.

- En términos de grupos etarios, los jóvenes y adultos mayores son los que tienen menor conectividad: el $42 \%$ de los menores de 25 años y el $54 \%$ de las personas mayores de 66 años no tienen conexión a Internet. Los grupos con menor conectividad son los de los niños de 5 a 12 años y el de los adultos mayores de 65 años, mientras que los más conectados son los grupos etarios de 21 a 25 años y de 26 a 65 años.

- Las bajas velocidades de conexión consolidan situaciones de exclusión ya que inhabilitan el uso de soluciones digitales de teletrabajo y educación en línea (véase el cuadro 1).

Cuadro 1 | Funcionalidades según velocidades de descarga de banda ancha

\begin{tabular}{lll}
\hline Baja & Media & Alta \\
\hline $\mathbf{5 , 5}$ Mbp/s & $\mathbf{1 8 , 5} \mathbf{M b p} / \mathbf{s}$ & Más de $\mathbf{2 5} \mathrm{Mbp} / \mathrm{s}$ \\
\hline $\begin{array}{l}\text { Permite utilizar funciones como correo } \\
\text { electrónico, vídeo básico y transmisión } \\
\text { directa de audio y vídeo a través } \\
\text { de Internet (streaming) }\end{array}$ & $\begin{array}{l}\text { Permite realizar simultáneamente } \\
\text { dos funciones básicas y una } \\
\text { actividad en línea de alta demanda }\end{array}$ & $\begin{array}{l}\text { Permite realizar simultáneamente } \\
\text { funciones básicas y funciones } \\
\text { de alta demanda }\end{array}$ \\
\hline $\begin{array}{l}\text { No permite el teletrabajo } \\
\text { No permite la educación en línea }\end{array}$ & $\begin{array}{l}\text { Permite el teletrabajo y la } \\
\text { educación en línea de manera } \\
\text { no simultánea }\end{array}$ & $\begin{array}{l}\text { Permite el teletrabajo } \\
\text { y la educación en línea } \\
\text { de manera simultánea }\end{array}$ \\
\hline
\end{tabular}

Fuente: Comisión Económica para América Latina y el Caribe (CEPAL), sobre la base de Comisión Federal de Comunicaciones (FCC), “Guía de velocidades de banda ancha", febrero de 2020 [en línea] https://www.fcc.gov/consumers/guides/guia-de-velocidades-de-banda-ancha. 
- Desde el comienzo de la crisis del COVID-19, la demanda de servicios de comunicación de banda ancha se ha incrementado vertiginosamente. El aumento del tráfico ha supuesto una mayor exigencia para la capacidad de las redes y su resiliencia, y en varios países ha disminuido la velocidad de descarga promedio de las redes durante los primeros meses de cuarentena, situación que, de acuerdo con los datos disponibles, se habría revertido.

- Pese a ello, a junio de 2020, en el $44 \%$ de los países de la región no se alcanzaba la velocidad de descarga que permite desarrollar varias actividades en línea simultáneamente: tienen una velocidad de conexión inferior a los $25 \mathrm{Mbps}$ (véase el gráfico 3). Las velocidades de descarga de alrededor de 18,5 Mbps permiten desarrollar simultáneamente dos actividades básicas, como la utilización del correo electrónico y la realización de una actividad de alta demanda, como hacer uso de vídeo o videoconferencia, lo que obliga a los usuarios a elegir entre la educación en línea y el teletrabajo. Cuando la velocidad de descarga es inferior a los 5,5 Mpbs, los usuarios pueden desarrollar solo actividades básicas y no pueden acceder al teletrabajo o la educación en línea.

Gráfico 3 América Latina y el Caribe (18 países)a: velocidades de descarga de la banda ancha móvil y fija, junio de 2020 (En porcentajes)

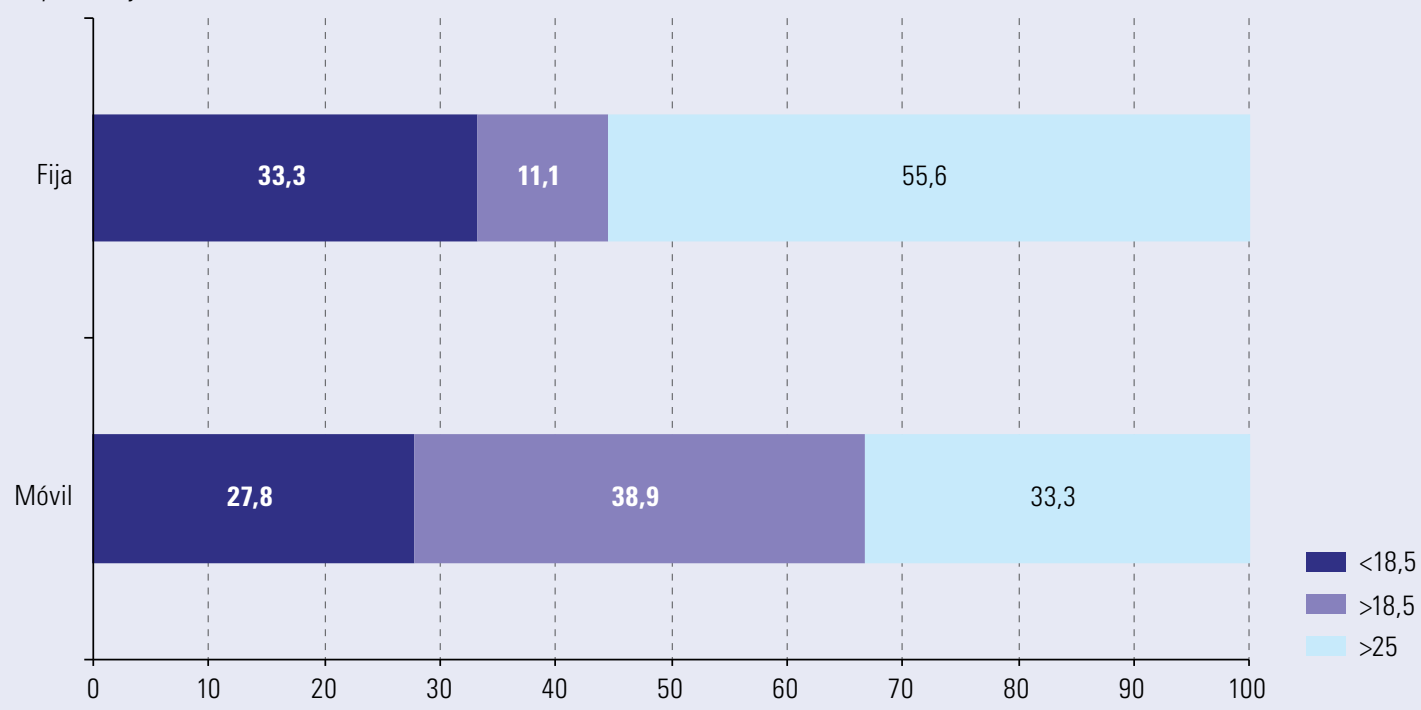

Fuente: Comisión Económica para América Latina y el Caribe (CEPAL), Observatorio Regional de Banda Ancha (ORBA), sobre la base de Ookla, "Speedtest Global Index" [en línea] http://www.speedtest.net/global-index.

a Argentina, Bolivia (Estado Plurinacional de), Brasil, Chile, Colombia, Costa Rica, Ecuador, El Salvador, Guatemala, Honduras, México, Nicaragua, Panamá, Paraguay, Perú, República Dominicana, Uruguay y Venezuela (República Bolivariana de).

- En el caso de la banda ancha móvil, el $67 \%$ de los países no cuenta con velocidades de descarga adecuadas para realizar actividades de alto consumo de datos de manera simultánea.

- Cabe mencionar que, en la región, la penetración de la banda ancha móvil es cinco veces mayor que la de banda ancha fija.

\section{El bajo ingreso de los hogares limita el acceso a Internet y las posibilidades de uso de aplicaciones móviles}

- En 11 países de la región, el porcentaje de hogares desconectados se ubica entre el $60 \%$ y el $85 \%$; en los países con mayores tasas de conexión se ubica en alrededor del $\mathbf{3 0} \%$.

- El costo del servicio de banda ancha móvil y fija para la población del primer quintil de ingresos llega al $14 \%$ y el $\mathbf{1 2} \%$ de su ingreso, respectivamente (véase el gráfico 4 ). Esos costos, en los casos más críticos, representan alrededor de seis veces el umbral de referencia del $2 \%$ del ingreso recomendado por la Comisión sobre la Banda Ancha para el Desarrollo Sostenible para clasificar un servicio de Internet como asequible. 
Gráfico 4 | América Latina (11 países): asequibilidad de Internet fija y móvil por quintil de ingreso, 2019 (En porcentajes del ingreso de los hogares)

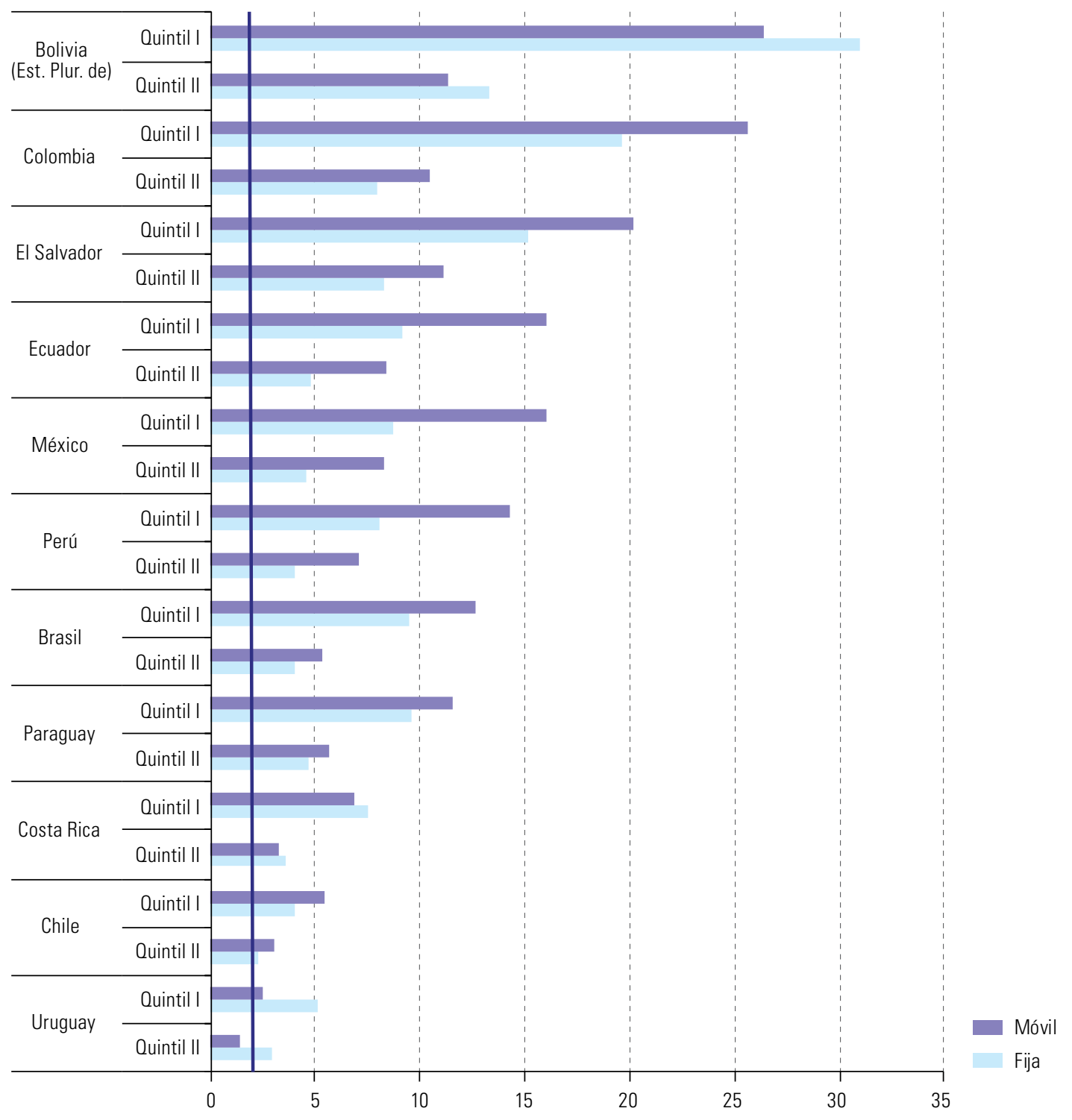

Fuente: Comisión Económica para América Latina y el Caribe (CEPAL), Observatorio Regional de Banda Ancha (ORBA), sobre la base de información del Banco de Datos de Encuestas de Hogares (BADEHOG).

Nota: El costo para calcular la asequibilidad de la banda ancha de Internet fija y móvil toma en consideración el costo promedio del acceso al servicio por país.

\section{a) Teletrabajo}

- El teletrabajo ha sido una herramienta fundamental para mantener en funcionamiento algunas empresas y evitar la proliferación de contagios del coronavirus. Antes de la pandemia, el 7,9\% de los trabajadores del mundo trabajaba permanentemente desde su hogar, principalmente en ocupaciones manufactureras y artesanales tradicionales, pero solo una minoría lo hacía mediante teletrabajo².

- La proporción de trabajo susceptible de realizarse a distancia varía entre países por razones estructurales. Las estructuras de los mercados laborales, las estructuras productivas, los niveles de informalidad y la calidad de la infraestructura digital juegan un papel fundamental.

- El porcentaje de puestos de trabajo que pueden migrar al teletrabajo está positivamente vinculado al nivel del PIB per cápita y a menores grados de informalidad (véase el gráfico 5).

\footnotetext{
Organización Internacional del Trabajo (OIT), "Working from home: estimating the worldwide potential", Policy Brief, abril de 2020.
} 
Gráfico 5 | Probabilidad de teletrabajar, PIB per cápita y nivel de informalidad, 2019 o último dato disponible (En porcentajes y dólares)

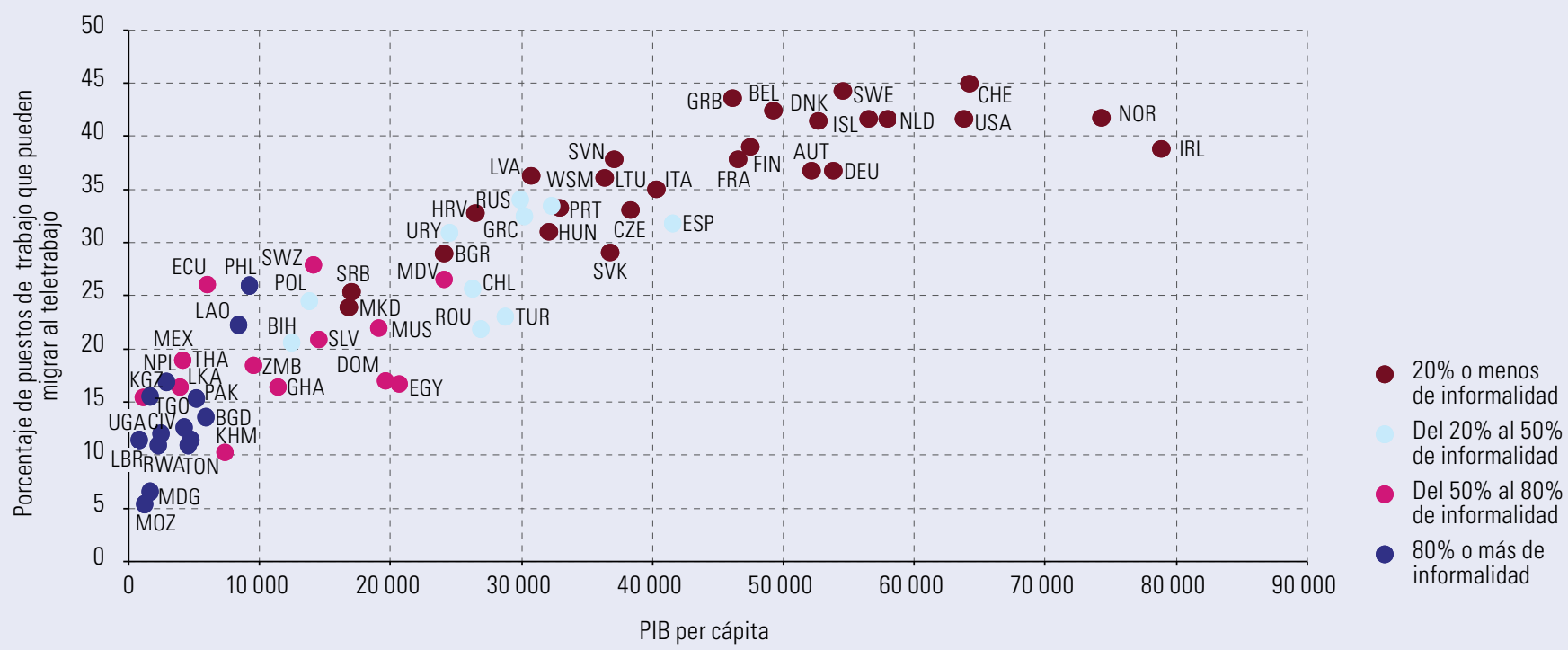

Fuente: Comisión Económica para América Latina y el Caribe (CEPAL), sobre la base de información del Banco de Datos de Encuestas de Hogares (BADEHOG): J. Dingel y B. Neiman, "How many jobs can be done at home?", NBER Working Paper, № 26948, Cambridge, Oficina Nacional de Investigaciones Económicas (NBER), 2020, y datos de la Organización Internacional del Trabajo (OIT).

- En Europa y los Estados Unidos, casi el $40 \%$ de los trabajadores puede trabajar desde su hogar, cifra que se reduce a menos del 15\% en algunos países de África. En el caso de América Latina y el Caribe, la Comisión Económica para América Latina y el Caribe (CEPAL) estima que alrededor del $\mathbf{2 1 , 3 \%}$ de los ocupados podría teletrabajar (véase el gráfico 6).

Gráfico 6 | América Latina y el Caribe: probabilidad de teletrabajar (En porcentajes)



Fuente: Comisión Económica para América Latina y el Caribe (CEPAL), sobre la base de información del Banco de Datos de Encuestas de Hogares (BADEHOG) y J. Dingel y B. Neiman, "How many jobs can be done at home?", NBER Working Paper, № 26948, Cambridge, Oficina Nacional de Investigaciones Económicas (NBER), 2020.

Nota: Las cifras del Ecuador, México, El Salvador, la República Dominicana y Chile se estiman sobre la base de las clasificaciones ocupacionales nacionales a cuatro dígitos. Las de los demás países se estiman sobre la base de Clasificación Internacional Uniforme de Ocupaciones (CIUO) de la Organización Internacional del Trabajo (OIT) a dos dígitos. Los datos corresponden a 2018 o al último año disponible.

- En la región, el porcentaje de ocupaciones en las que se podría teletrabajar está condicionado por los elevados niveles de informalidad, que en 2018 alcanzaban más del $\mathbf{5 0} \%$ del empleo total. La mayoría del empleo informal se concentra en sectores que necesitan interacción física, por lo que no se puede desarrollar en forma remota. 
- Las características de la estructura productiva de la región limitan el porcentaje de ocupaciones que pueden migrar al teletrabajo debido a la alta concentración de trabajadores en actividades que necesitan interacción social y presencia física. A nivel sectorial, la probabilidad de teletrabajar es mayor del $\mathbf{8 0} \%$ en los servicios profesionales, científicos y técnicos, la educación, las finanzas y los seguros. En los países de la región, estos sectores representan menos del $\mathbf{2 0} \%$ de los ocupados. Por el contrario, las probabilidades de teletrabajar de los ocupados en el comercio mayorista y minorista y la agricultura son del $15 \%$ y el $1 \%$ respectivamente (véase el gráfico 7 ).

Gráfico 7 | Probabilidad de teletrabajar por sector de actividad económica ${ }^{a}$ (En porcentajes)

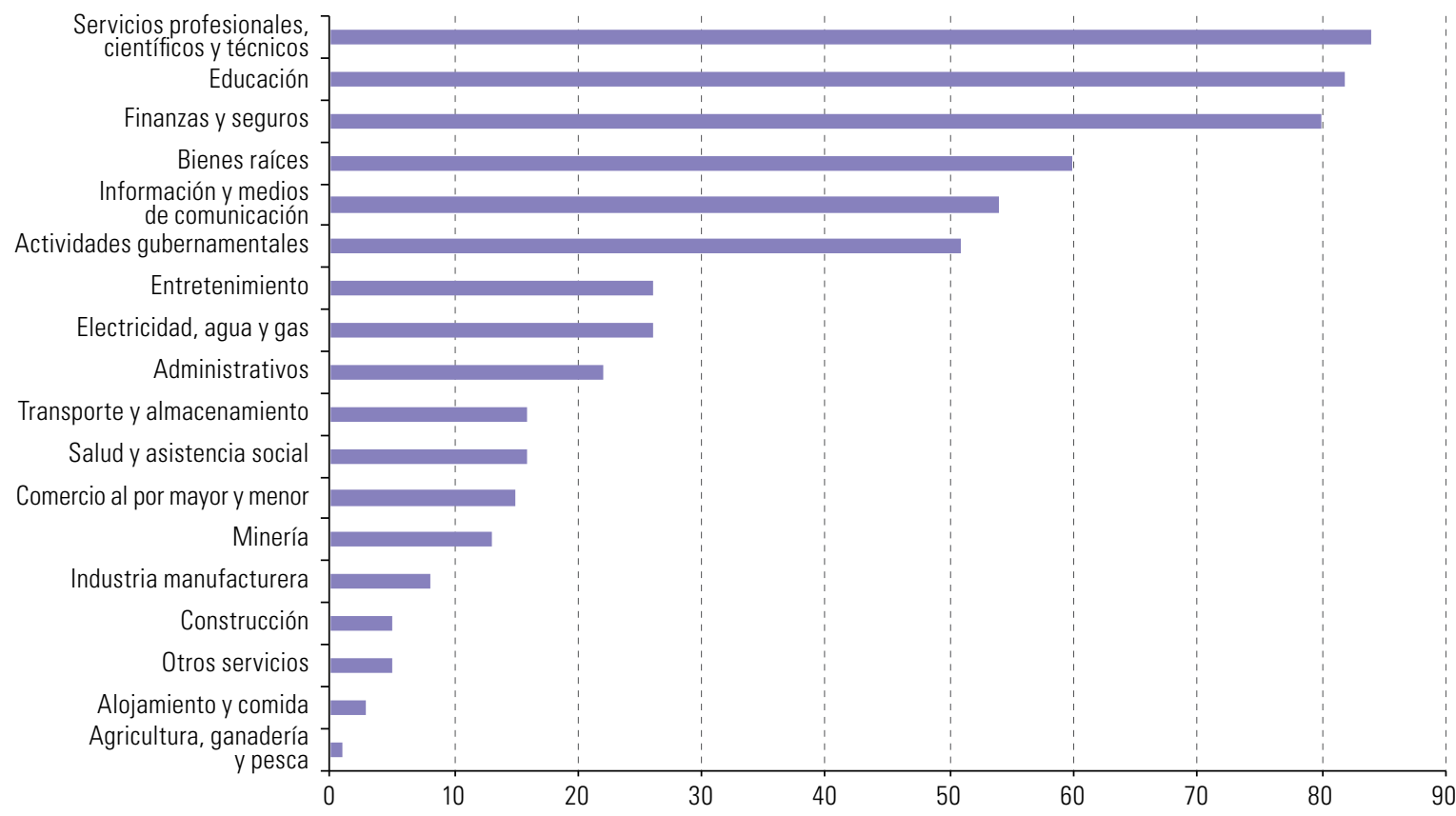

Fuente: Comisión Económica para América Latina y el Caribe (CEPAL), sobre la base de información del Banco de Datos de Encuestas de Hogares (BADEHOG) y J. Dingel y B. Neiman, "How many jobs can be done at home?", NBER Working Paper, N 26948, Cambridge, Oficina Nacional de Investigaciones Económicas (NBER), 2020.

a Promedios ponderados de: Chile, Ecuador, El Salvador, México, República Dominicana y Uruguay.

\section{- Otros factores condicionantes son la infraestructura digital, el nivel de digitalización de las empresas y las habilidades digitales.}

- En el gráfico 8 se muestra el porcentaje de ocupados en trabajos que se pueden desarrollar telemáticamente y que no pueden trabajar desde su casa debido al nivel de conectividad del país. Así, por ejemplo, en la República Dominicana, la baja conectividad reduce el porcentaje de ocupados que podrían teletrabajar en 11 puntos porcentuales. En países con mejor conectividad, como Chile y el Uruguay, el porcentaje de trabajadores que no podría trabajar de forma remota por esta causa se reduce 1 punto y 3 puntos porcentuales, respectivamente.

- Si bien la digitalización ha tenido un papel fundamental en el mantenimiento de algunas actividades productivas durante los períodos de cuarentena, preocupa que se profundice la desigualdad que caracteriza a los mercados laborales en América Latina y el Caribe.

- En ausencia de políticas de apoyo a los trabajadores más vulnerables en un contexto de grandes desigualdades en el acceso a las herramientas tecnológicas, elevada heterogeneidad en las capacidades de los trabajadores de diversos niveles de ingreso y una estructura productiva concentrada en actividades de bajo valor agregado, el teletrabajo profundiza las desigualdades.

- En la mayoría de las ocupaciones que se pueden desarrollar de manera remota los trabajadores tiene un mayor nivel de formación y, en promedio, perciben salarios mayores que los de las actividades que no se pueden hacer en forma remota. 
Gráfico 8 América Latina (6 países): ocupados en trabajos que se pueden desarrollar telemáticamente y que no pueden trabajar desde su casa debido al nivel de conectividad del país, 2018 o último dato disponible

(En puntos porcentuales)

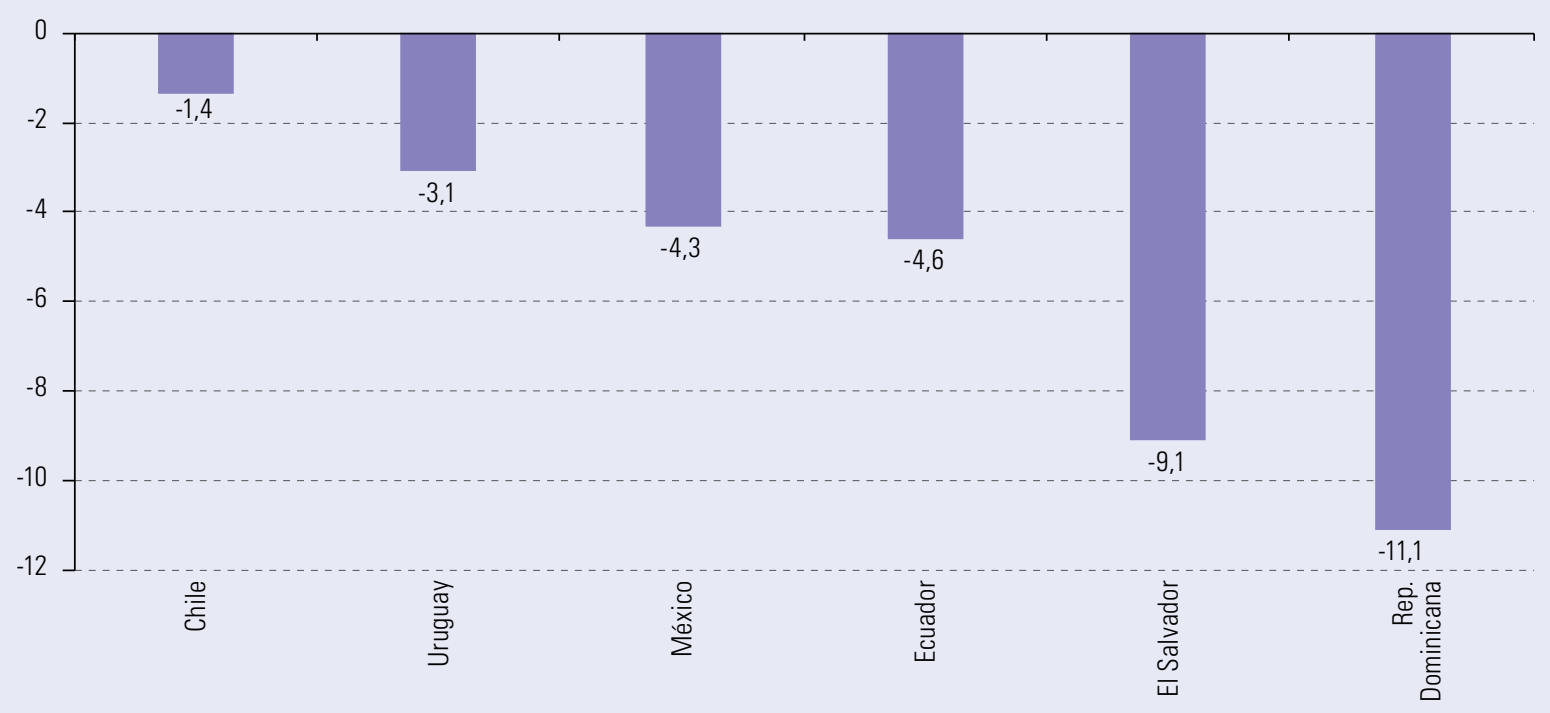

Fuente: Comisión Económica para América Latina y el Caribe (CEPAL), sobre la base de información del Banco de Datos de Encuestas de Hogares (BADEHOG) y J. Dingel y B. Neiman, "How many jobs can be done at home?", NBER Working Paper, №26948, Cambridge, Oficina Nacional de Investigaciones Económicas (NBER), 2020.

- En el gráfico 9 se muestra que en los quintiles I, II y III de salarios, más del $\mathbf{8 0 \%}$ de los ocupados no puede teletrabajar, mientras que en los quintiles IV y V, más del $50 \%$ de los ocupados puede hacerlo.

Gráfico 9 | América Latina (5 países)a: ocupados según posibilidad de teletrabajar y quintil de salario promedio de las ocupaciones (En porcentajes)

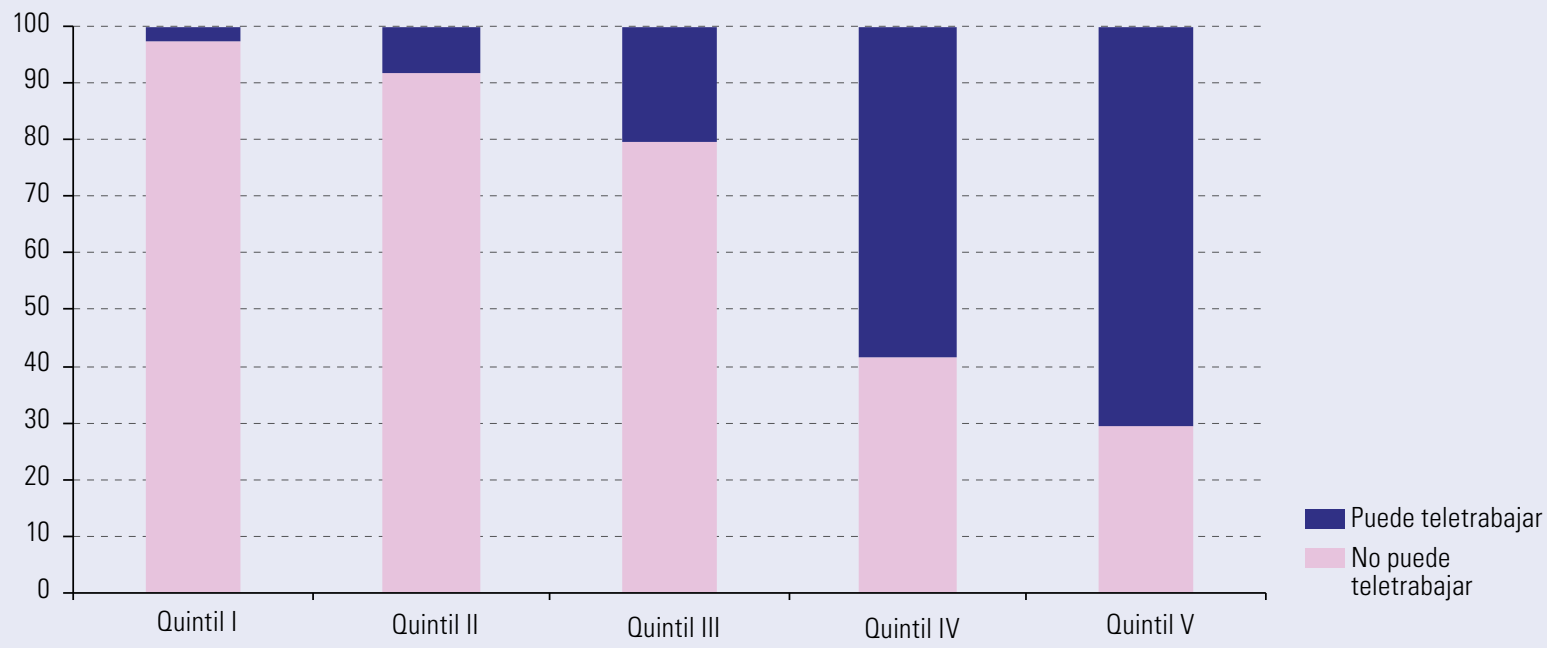

Fuente: Comisión Económica para América Latina y el Caribe (CEPAL), sobre la base de información del Banco de Datos de Encuestas de Hogares (BADEHOG)

a Promedio ponderado de: Chile, Ecuador, El Salvador, México, República Dominicana y Uruguay.

- Cuanto más duren las medidas de cuarentena y la suspensión de la actividad económica mayores serán las consecuencias para los trabajadores que no pueden teletrabajar, lo que aumenta las vulnerabilidades y desigualdades.

- Durante la crisis, quienes desarrollan actividades básicas o manuales y no pueden migrar al teletrabajo están más expuestos a reducciones salariales o pérdidas de empleo, mientras que los trabajadores más calificados pueden mantener sus ingresos. 
- Para mitigar los efectos de las medidas de cuarentena, las fases de reapertura de la economía deben tomar en cuenta planes de retorno a los lugares de trabajo que privilegien las ocupaciones de salarios más bajos que no se pueden desarrollar desde el hogar.

\section{b) Educación en línea}

- Debido a la suspensión de las clases presenciales, los países de la región han desarrollado estrategias para sostener actividades educativas a distancia.

- Los países que contaban con plataformas virtuales de contenidos educativos pusieron el foco en su adecuación y actualización. Los demás países pusieron en línea nuevas plataformas virtuales, en algunos casos en cooperación con empresas como Microsoft, Cisco y Google, y con organismos multilaterales. En la mayoría de los casos, las plataformas de contenido se complementaron con soluciones de aulas virtuales.

- El uso de soluciones de educación en línea solo es posible para quienes cuentan con conexión a Internet y dispositivos de acceso. El 46\% de los niños y niñas de entre 5 y 12 años de la región vive en hogares que no están conectados a Internet. En los países respecto de los que se cuenta con información, esto implica la exclusión de más de $\mathbf{3 2}$ millones de niños y niñas (véase el gráfico 10).

Gráfico 10 | América Latina (13 países): niños en hogares sin acceso a Internet, por quintil de ingreso (En porcentajes)

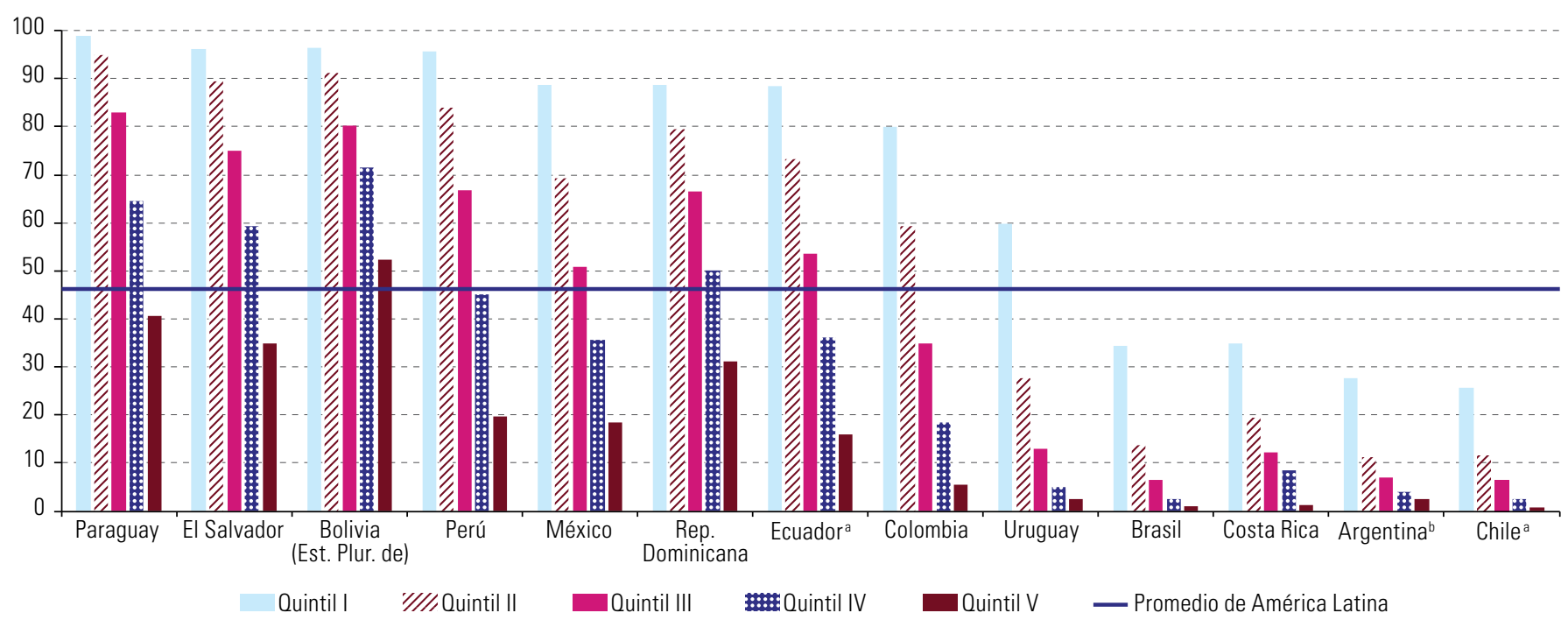

Fuente:Comisión Económica para América Latina y el Caribe (CEPAL), sobre la base de información del Banco de Datos de Encuestas de Hogares (BADEHOG)

Nota: En la encuesta en la que se basan los datos, "hogares con acceso a Internet" se refiere a los hogares en que Internet está generalmente disponible para su utilización por todos los miembros del hogar en cualquier momento; la conexión y los dispositivos pueden ser propiedad de la familia o no, pero se deben considerar los activos del hogar; la conexión a Internet en el hogar debía estar funcionando en el momento de la encuesta. El cálculo se hace sobre el total de niños de entre 5 y 12 años en cada quintil de ingreso de cada país.

a La información corresponde a 2017

b Incluye solo la zonas urbanas.

- En Bolivia (Estado Plurinacional de), El Salvador, el Paraguay y el Perú, más del $90 \%$ de los niños y niñas de los hogares más pobres viven en hogares sin conexión a Internet.

- En los países que presentan mejores indicadores de conectividad, alrededor del $30 \%$ de estos niños y niñas no cuentan con conexión a Internet en su hogar.

- El número promedio de niños de hogares de menores ingresos en los países de la región cuadruplica el número promedio de niños de los hogares de mayores ingresos, lo que dificulta el acceso a Internet de los primeros, pues es necesario contar con más dispositivos en un mismo hogar para la conexión de cada uno de ellos.

- El acceso de los hogares a los dispositivos digitales es también desigual en la región, especialmente entre los distintos niveles socioeconómicos y culturales. Mientras que entre 
el $\mathbf{7 0} \%$ y el $\mathbf{8 0} \%$ de los estudiantes de los niveles socioeconómicos más altos (cuarto cuartil) tienen computadoras portátiles en sus hogares, solo entre el $10 \%$ y el $20 \%$ de los estudiantes pertenecientes a los quintiles de menores ingresos (primer cuartil) cuentan con estos dispositivos (véase el gráfico 11).

Gráfico 11 | América Latina (7 países): estudiantes de 15 años que cuentan con dispositivos digitales en el hogar, por tipo de dispositivo y cuartil socioeconómico, 2018

(En porcentajes)

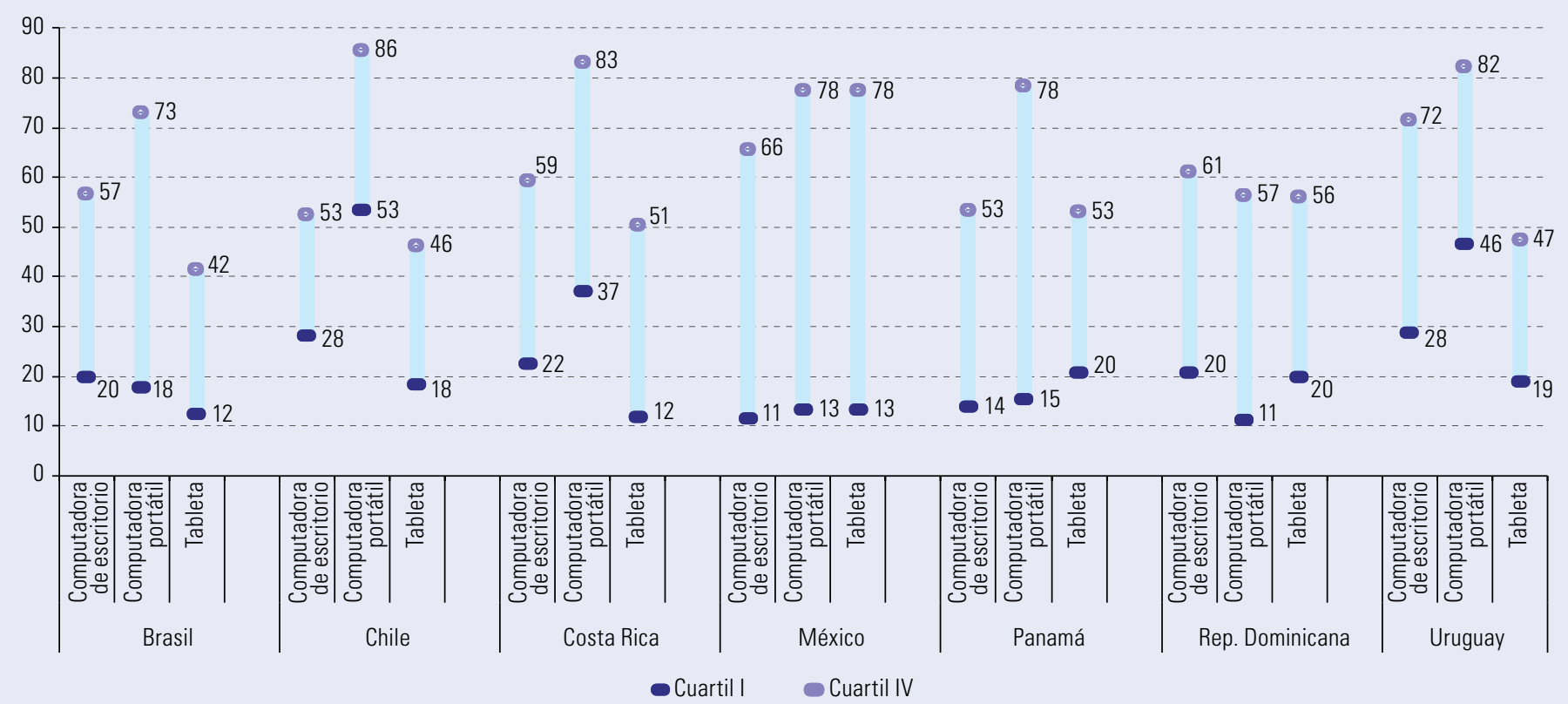

Fuente: Comisión Económica para América Latina y el Caribe (CEPAL)/Organización de las Naciones Unidas para la Educación, la Ciencia y la Cultura (UNESCO), "La educación en tiempos de la pandemia de COVID-19", Informe COVID-19, Santiago, 20 de agosto de 2020.

- La diferencia entre los estratos económicos más altos y más bajos condiciona el derecho a la educación y profundiza las desigualdades socioeconómicas.

- Para garantizar una educación inclusiva y equitativa y promover oportunidades de aprendizaje a lo largo de todo el ciclo educativo, se debe aumentar no solo la conectividad y la infraestructura digital sino también las habilidades digitales de maestros y profesores, así como la adecuación de los contenidos educativos al ámbito digital. También se recomienda explorar el uso de herramientas digitales de educación específicamente diseñadas para un contexto de baja conectividad y el uso dispositivos móviles básicos.

- Las respuestas en materia educativa de los países de la región frente a la pandemia de COVID-19 han sido diversas.

- En algunos casos, se ha considerado un abordaje integral de la emergencia, mediante el diseño de un plan exhaustivo articulado con otras medidas económicas, sociales y de salud.

- En otros, hay iniciativas puntuales que orientan la gestión de los centros educativos. En esos casos, las respuestas de planificación son menos transversales y su contenido está enfocado en mantener la continuidad pedagógica y la comunicación con las familias. La mayor parte de estas intervenciones se difunden mediante guías, protocolos de intervención, resoluciones o noticias publicadas en las páginas web de los ministerios correspondientes.

- Generar una propuesta educativa a distancia más inclusiva supone considerar el contexto y las posibilidades que ofrece la infraestructura digital y el acceso a servicios de Internet y a dispositivos.

- De los 29 países analizados, 23 complementan el aprendizaje en línea con estrategias que se apoyan en la televisión y la radio. Por otro lado, solamente 8 países dispusieron la entrega de dispositivos a la población sin acceso a estos (véase el gráfico 12).

- En 24 países se implementaron estrategias de aprendizaje con recursos impresos para la población sin acceso a medios digitales y audiovisuales. 
Gráfico 12 | América Latina y el Caribe (29 países)a: estrategias para la educación continua a distancia, por modalidad de aprendizaje, 2020

(En número de países que las aplican)

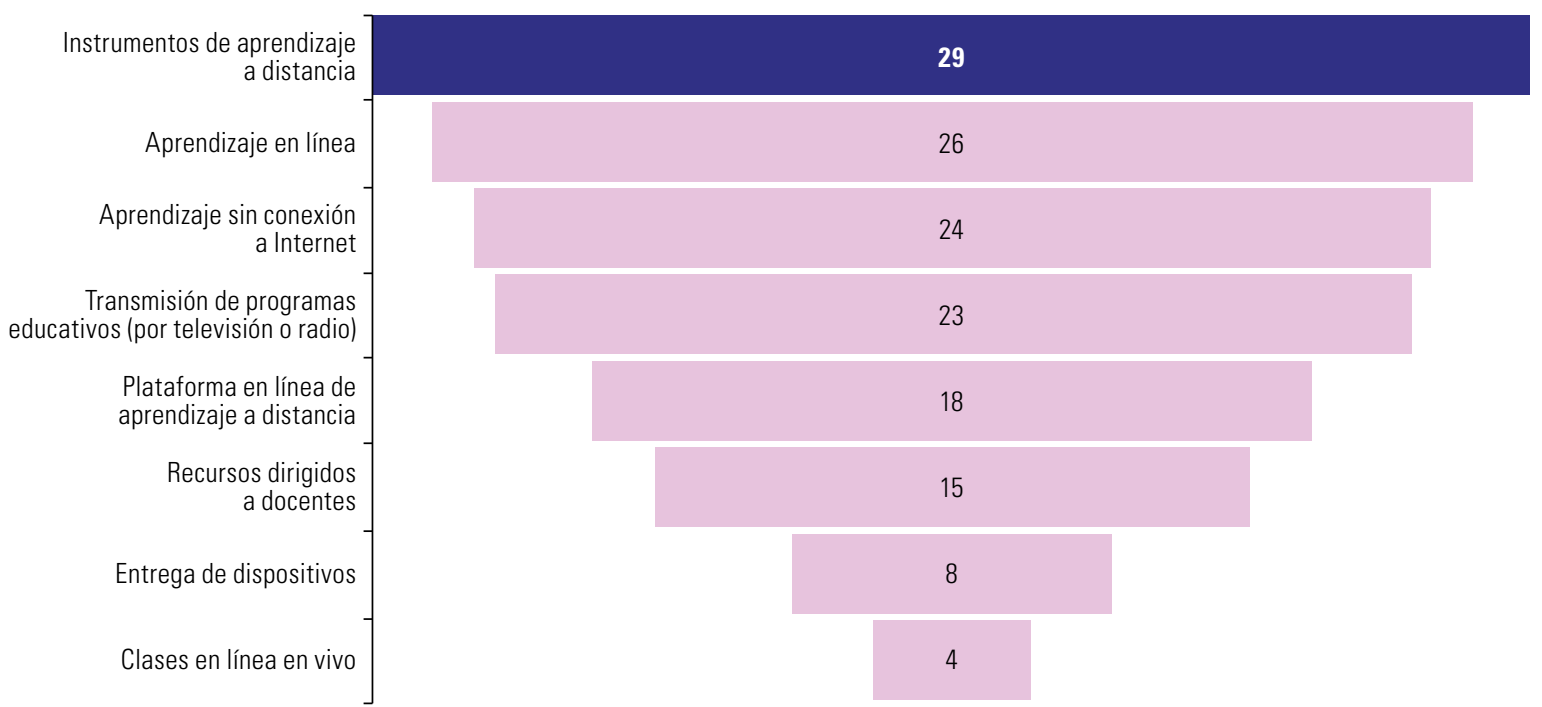

Fuente: Comisión Económica para América Latina y el Caribe (CEPAL)/Organización de las Naciones Unidas para la Educación, la Ciencia y la Cultura (UNESCO), “La educación en tiempos de la pandemia de COVID-19", Informe COVID-19, Santiago, 20 de agosto de 2020.

a Argentina, Bahamas, Barbados, Belice, Bolivia (Estado Plurinacional de), Brasil, Chile, Colombia, Costa Rica, Cuba, Ecuador, El Salvador, Granada, Guatemala, Guyana, Haití, Honduras, Jamaica, México, Nicaragua, Panamá, Paraguay, Perú, República Dominicana, Santa Lucía, Suriname, Trinidad y Tabago, Uruguay y Venezuela (República Bolivariana de).

\section{c) Servicios de salud electrónica}

- La prestación de servicios de salud electrónica cambia los modelos de atención. Sus beneficios son múltiples: mejora el acceso a los servicios, su eficiencia y la calidad de su prestación, reduce los costos y aumenta la capacidad de prevención de enfermedades.

- Contribuye además a descongestionar los centros de salud y hospitales, frenar la transmisión del coronavirus y aplanar las curvas epidémicas y de contagio. Los síntomas del COVID-19 y la recuperación del paciente se pueden monitorear a través de llamadas telefónicas o videollamadas, manteniéndose así a los pacientes de bajo riesgo y con síntomas leves en sus hogares, lo que reduce la probabilidad de transmitir el virus. Esta modalidad de prestación de servicios de salud en línea requiere de políticas que prevean la sanción de marcos legales para su implementación, la digitalización de la información médica, la interoperabilidad y la atención de aspectos como la privacidad y la seguridad de los datos.

- En 2015 , el $56 \%$ de los países de la región contaba con una política o estrategia nacional de prestación de servicios de salud en forma electrónica.

- Sin embargo, solo un $\mathbf{3 8} \%$ de esos países contaba con una regulación específica para el intercambio de datos digitales entre los servicios de salud, lo que pone de manifiesto la necesidad de un componente central del marco regulatorio habilitante (véase el cuadro 2).

- Más allá de aumentar la demanda de los servicios de salud a distancia, la pandemia ha puesto de relieve algunas debilidades estructurales de los sistemas médicos y de salud electrónica. Los costos y la incertidumbre respecto de los reembolsos son barreras al uso de la salud electrónica; frecuentemente los pacientes y los proveedores de atención médica carecen de información sobre los pagos y la cobertura de los seguros.

- Otras debilidades tienen que ver con la edad y el nivel de formación de los pacientes: los menos capacitados digitalmente (por ejemplo, las personas mayores) son más vulnerables y tienen menor capacidad para beneficiarse de las soluciones de salud electrónica. 
Cuadro 2 | América Latina y el Caribe (16 países): marcos normativos para la prestación de servicios de salud en forma electrónica, 2015


Fuente: Comisión Económica para América Latina y el Caribe (CEPAL), sobre la base de datos de Organización Mundial de la Salud (OMS), Observatorio Mundial de Cibersalud.

- Los Gobiernos de la región han desarrollado aplicaciones móviles para minimizar el contacto físico entre pacientes y proveedores de atención médica y difundir información esencial sobre formas de prevención del contagio del coronavirus y noticias sobre la pandemia.

- Gran parte de esas aplicaciones permiten acceder a información sobre la ubicación de los establecimientos de salud y los autodiagnósticos (véase el cuadro 3). En algunos casos, luego de un primer autodiagnóstico, la aplicación deriva al usuario directamente a un centro de atención sanitario, como es el caso de la aplicación CuidAr COVID-19 del Ministerio de Salud de la Argentina.

- Una funcionalidad que solamente se encuentra en las aplicaciones desarrolladas por los Gobiernos del Ecuador y el Perú es el agendamiento de citas médicas de forma priorizada mediante una aplicación, lo que se conoce como triaje virtual.

- Los sistemas de rastreo de contactos (contact tracing) no son utilizados ampliamente mediante las aplicaciones móviles. En la región, solamente las aplicaciones del Perú y el Uruguay proporcionan información sobre posibles contactos con personas infectadas o cuentan con algún tipo de notificación de exposición.

- Otra función poco utilizada es la comunicación con personas en cuarentena y el pasaporte sanitario o certificado de circulación. 




Fuente: Comisión Económica para América Latina y el Caribe (CEPAL).

\section{d) Comercio electrónico y digitalización de empresas}

- Presencia de las empresas en Internet para la recuperación. A medida que se extiende la duración de la pandemia, las empresas perciben las oportunidades de tener una presencia en línea para llegar a los consumidores, lo que se observa en el significativo aumento de la cantidad de sitios web empresariales en el Brasil, Chile, Colombia y México en marzo, abril y mayo de 2020 en comparación con el año anterior. Entre abril y marzo de 2020, el incremento del número de estos sitios fue del $\mathbf{8 0 0} \%$ en Colombia y México, y alrededor del $\mathbf{3 6 0} \%$ en el Brasil y Chile (véase el gráfico 13).

Gráfico 13 | América Latina (4 países): nuevos sitios web empresariales registrados al mes y crecimiento mensual, 2018-2020 (En número de sitios y porcentajes)

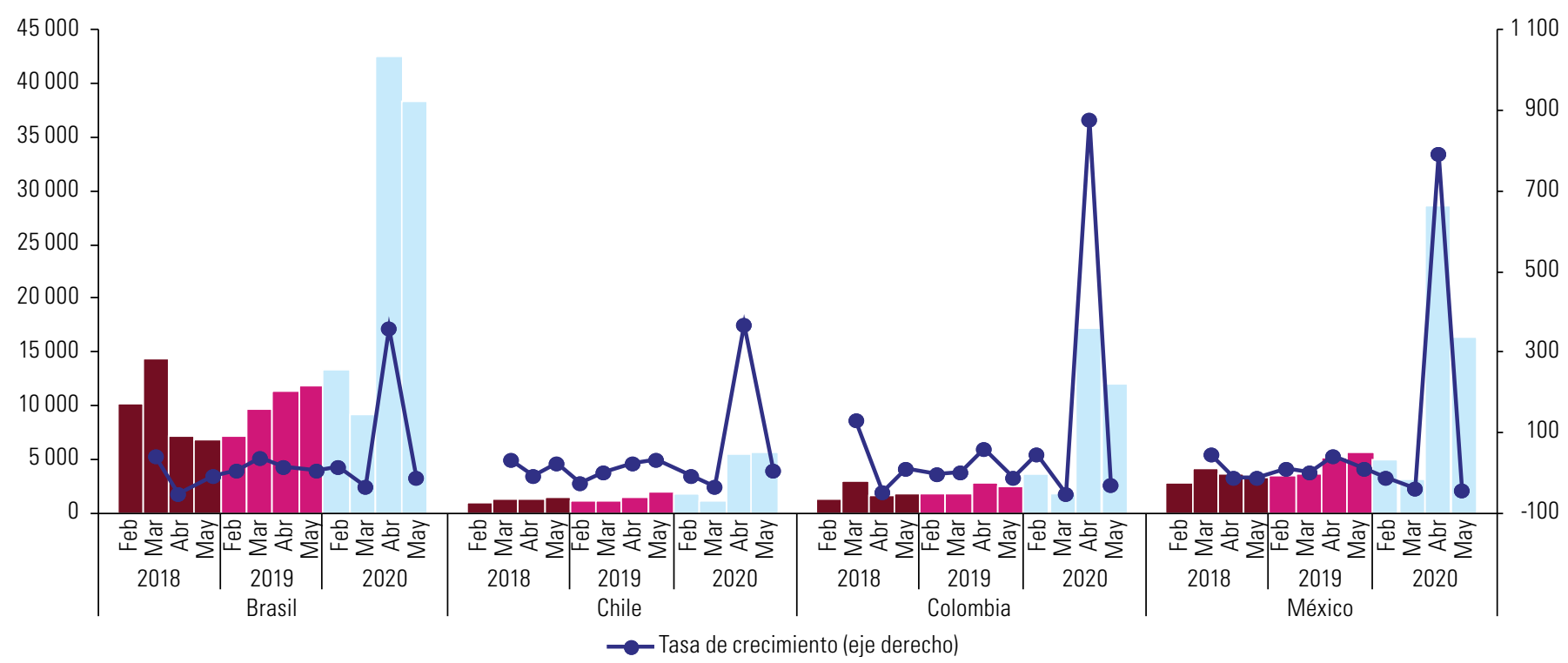

Fuente: Comisión Económica para América Latina y el Caribe (CEPAL), proyecto "Grandes datos para la economía digital en América Latina y el Caribe", 2020. 
- El comercio electrónico se vuelve esencial. Los mayores incrementos de la presencia en línea se registran en los sitios empresariales de tipo transaccional (presencia activa) y los sitios de plataforma de comercio electrónico. En el Brasil y México, el número de sitios nuevos de comercio electrónico aumentó más del $\mathbf{4 5 0} \%$ en abril de 2020 en comparación con el mismo mes de 2019. En tanto, los sitios con presencia activa en Colombia y México aumentaron cerca del $500 \%$ en el mismo período (véase el gráfico 14).

Gráfico 14 | América Latina (4 países): crecimiento interanual de sitios web empresariales, por tipo, 2019-2020 (En porcentajes)

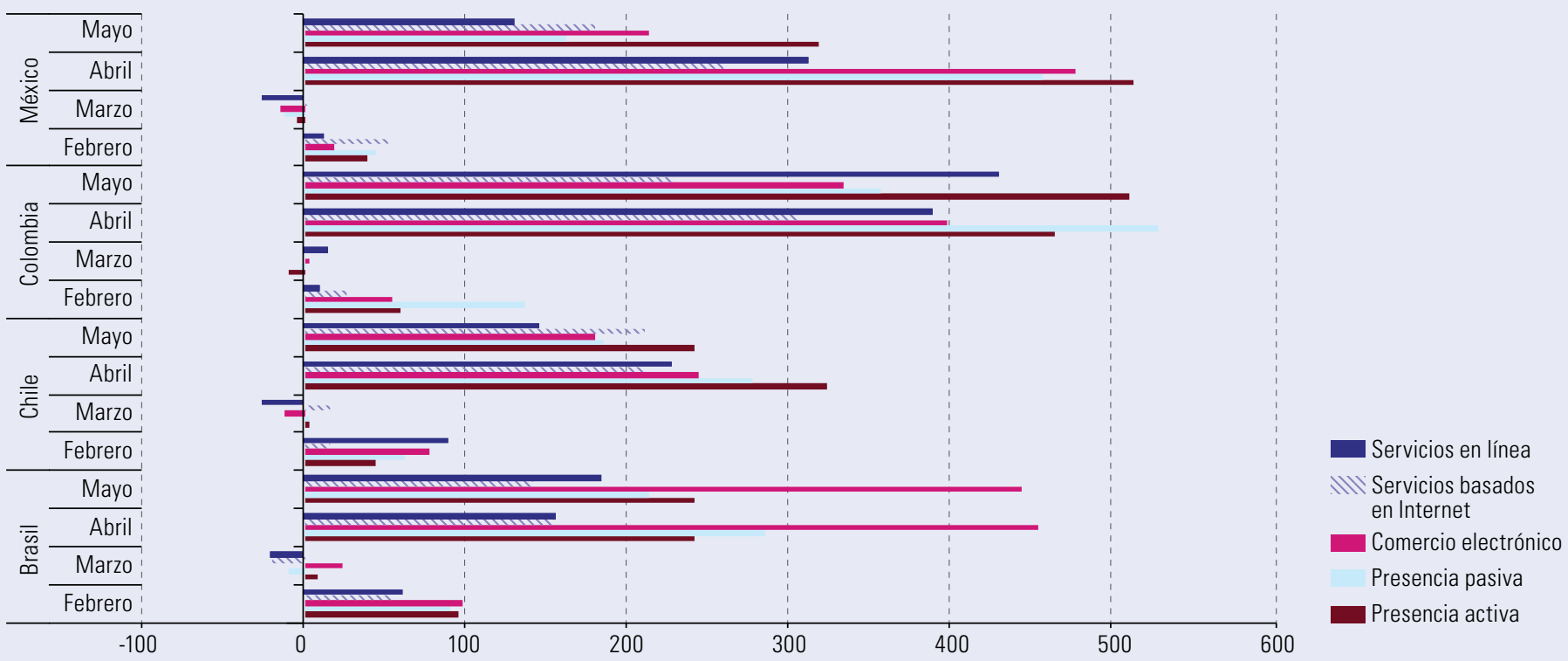

Fuente: Comisión Económica para América Latina y el Caribe (CEPAL), proyecto "Grandes datos para la economía digital en América Latina y el Caribe", sobre la base de Dataprovider.com, 2020 [en línea] https://www.dataprovider.com.

Nota: Categorías definidas según la forma en que las empresas generan ingresos basadas en el uso de Internet (Oostrom y otros, "Measuring the internet economy in the Netherlands: a big data analysis", Discussion Paper, La Haya, Oficina Central de Estadísticas de los Países Bajos, 2016. Presencia pasiva en línea: solo brindan información o propaganda de sus actividades comerciales. Presencia activa en línea: sitios web que permiten la interacción con los clientes como apoyo a las actividades comerciales principales (como venta en línea y atención al consumidor). Comercio electrónico: tiendas en línea sin presencia física que generan sus ventas a través de Internet. Servicios en línea: provisión de servicios en línea que existirían incluso sin Internet (como alojamiento y turismo). Servicios relacionados con la tecnología de la información y las comunicaciones basada en Internet: actividades que existen gracias a Internet (diseño web, servicios de alojamiento, servicios en la nube y desarrollo de aplicaciones).

- En junio de 2020, la presencia en línea de empresas de comercio minorista aumentó el $431 \%$ con respecto a junio de 2019; el incremento en el caso de restaurantes y servicios de entrega de comida fue del $331 \%$ y en el de servicios empresariales, el $311 \%$.

- Entre enero y junio de 2020, el análisis de los sitios web empresariales muestra un cambio en el tipo de presencia en línea. En los primeros seis meses de 2020, en el Brasil, Chile, Colombia y México se observa que el $20 \%$ de los cambios en los sitios web existentes corresponde a transición a sitios de tipo transaccional.

- La capacidad de entrega de los productos es fundamental. Los servicios de entrega han aumentado su relevancia ya que permiten disminuir la concurrencia masiva a supermercados, almacenes y tiendas, y permiten mantener la actividad de algunos locales comerciales.

- Entre el primer y segundo trimestre de 2020, la actividad de los servicios de entrega aumentó un $\mathbf{1 5 7 \%}$ según el tráfico registrados en los sitios web y plataformas de este rubro. En la Argentina, Chile y Colombia, a pesar de las medidas de cuarentena, se ha autorizado su utilización por ser esenciales para la entrega de alimentos. Las plataformas en línea de entrega han registrado una mayor demanda de sus servicios; en marzo, casi el $\mathbf{1 0 0} \%$ de los pedidos se concentraba en el rubro de alimentos y farmacias. Sin embargo, para los negocios pequeños, las comisiones por el servicio del orden del $20 \%$ o más pueden resultar un obstáculo para el uso de esas plataformas.

- La presencia en línea se ha vuelto particularmente importante para el comercio y los servicios empresariales, profesionales, de salud y de educación. 
- Durante la pandemia, las plataformas de comercio electrónico y entrega han cobrado un protagonismo inusitado. El aumento exponencial en el uso de estas plataformas, que al parecer será irreversible, plantea a los países la necesidad de fortalecer sus marcos regulatorios y normativos, así como las políticas de competencia para evitar abusos de poder de mercado que se podrían derivar del aumento de la concentración; por ejemplo, el cobro excesivo por los servicios.

- Por su parte, la digitalización de los procesos productivos se encuentra muy rezagada en la región. Si bien las empresas tienen un alto nivel de conectividad (cercana al $90 \%$ ) y el $80 \%$ utiliza la banca electrónica, el uso de las tecnologías digitales en los procesos de gestión —utilización de Internet en la cadena de aprovisionamiento, procesamiento, manufactura, operaciones y canales de distribución - se encuentra muy rezagada en comparación con países más desarrollados. Mientras el $\mathbf{7 0} \%$ de las empresas de los países de la Organización de Cooperación y Desarrollo Económicos (OCDE) utilizan Internet en su cadena de aprovisionamiento, en algunos países de América Latina esa cifra es de solo el $\mathbf{3 7 \%}$ (véase el gráfico 15).

Gráfico 15 | Brasil, Chile y Colombia: digitalización de los procesos productivos, 2018 (En porcentajes)

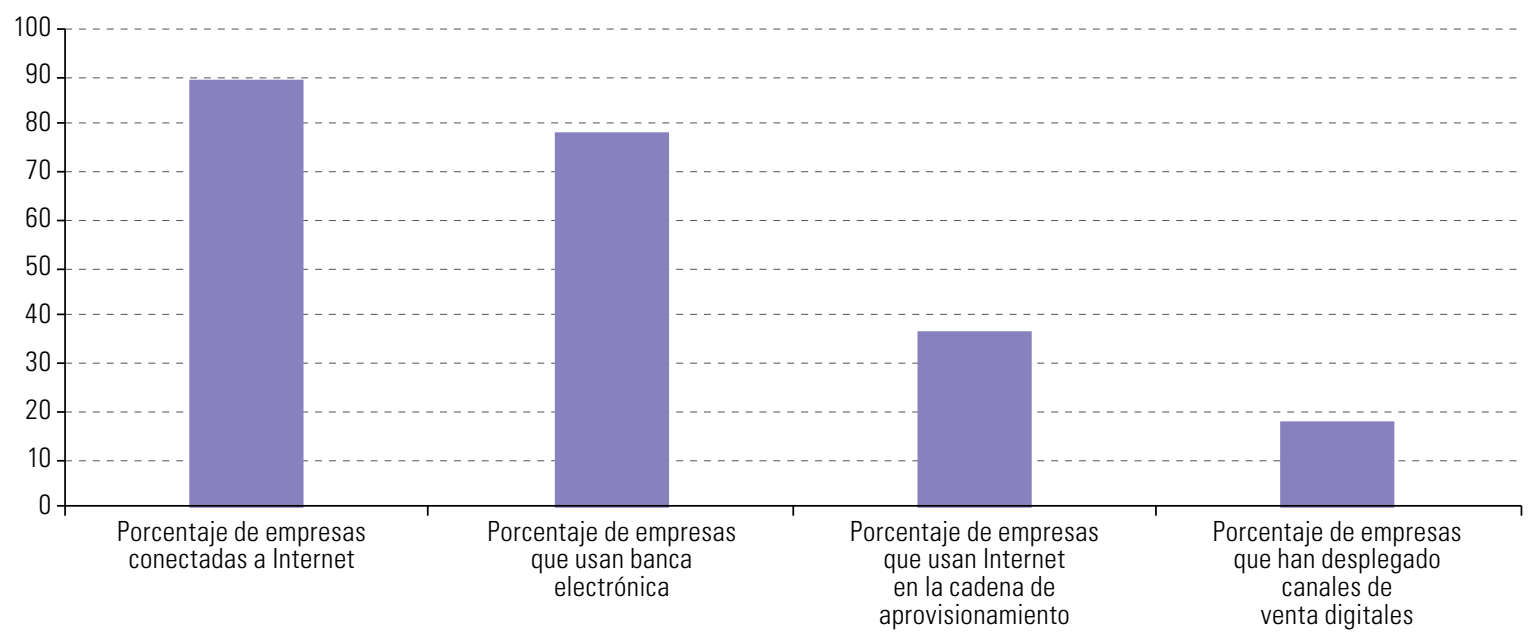

Fuente: Comisión Económica para América Latina y el Caribe (CEPAL), sobre la base de datos de las encuestas de tecnologías de la información y las comunicaciones (TIC) en empresas del Brasil, Chile y Colombia.

- Ante el avance de la transformación digital, la capacidad de los países para mitigar los riesgos y desafíos de la pandemia, responder a nuevas crisis y capitalizar las oportunidades de producción dependerá del grado de preparación que tengan para la producción del futuro.

- La estructura de la producción — en especial, la complejidad y la escala-y los factores impulsores de la producción — capacidades tecnológicas y de innovación, habilidades y capital humano, comercio e inversión, marco institucional y recursos sostenibles, entre otros- determinan el posicionamiento de los países con respecto a la manufactura avanzada o industria 4.0 (véase el gráfico 16).

- Sobre la base de ese análisis de posicionamiento, se identifican tres conjuntos de países: i) los muy bien posicionados para apropiarse del máximo beneficio de estas tecnologías, categoría en la que se encuentran los países desarrollados y algunos países del sudeste asiático; ii) países en una posición intermedia, como México, que cuentan con una estructura productiva que les permitiría explotar de mejor manera el potencial de las tecnologías digitales, pero carecen de los factores adecuados para hacerlo, como la capacidad e innovación y el capital humano, y iii) la mayor parte de los países de América Latina y el Caribe, que se encuentran en una posición de escaso acceso a las nuevas tecnologías y en situación de gran riesgo ante los efectos del avance tecnológico. 
Gráfico 16 | Países seleccionados: preparación para la producción del futuro

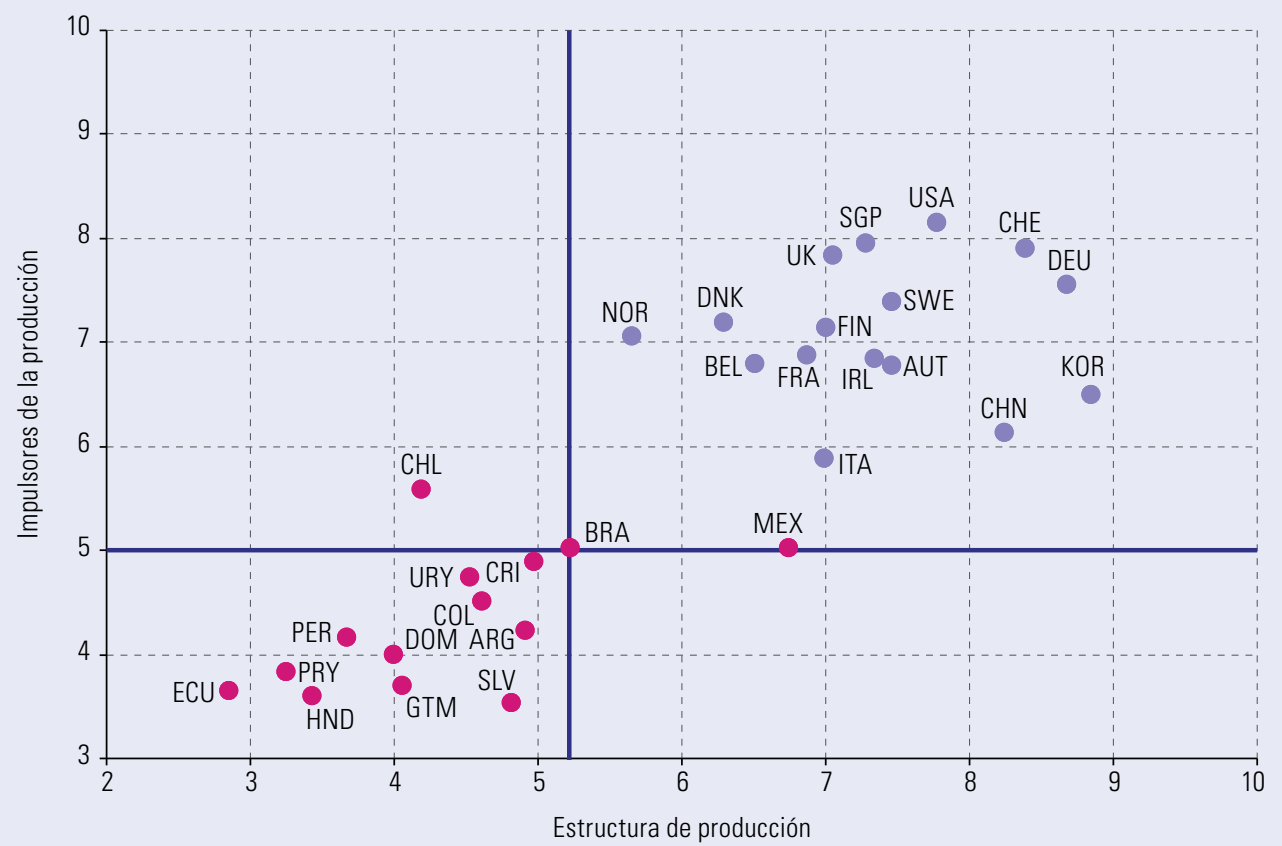

Fuente: Comisión Económica para América Latina y el Caribe (CEPAL), sobre la base de K. Schwab (ed.), The Global Competitiveness Report 2018, Ginebra, Foro Económico Mundial, 2018.

- Uno de los principales desafíos para avanzar en el proceso de recuperación luego de la pandemia es contar con las capacidades de innovación y el capital humano para desarrollar nuevas industrias y sectores y aumentar el valor agregado.

- La escasa formación, la limitada incorporación de las tecnologías digitales en los procesos formativos y los bajos niveles de inversión en innovación son los principales problemas que enfrenta la región. Mientras que la tasa bruta de matriculación en la educación terciaria en América Latina y el Caribe es del 50\%, en los países de la OCDE alcanza el 74\%; el coeficiente de alumnos por computadora es de 42 y 8; la cifra de patentes concedidas por millón de habitantes es de 1 y 211, y el gasto en investigación y desarrollo es del 0,67\% y el 2,15\% del PIB, todo ello respectivamente.

\section{El COVID-19 como acelerador de la discusión sobre la protección de los datos y la privacidad}

- La pandemia agrega una tensión adicional a la discusión sobre la gobernanza de Internet, ya que ha hecho necesario un despliegue total y coordinado de las tecnologías de la información, lo que genera grandes cantidades de datos a partir de las acciones coordinadas de las autoridades, los centros de salud e investigación y la población.

- Las amenazas que representa para la seguridad el despliegue masivo de datos sensibles son un nuevo tema de conflicto entre las autoridades y las empresas administradoras de datos.

- El estado de emergencia provocado por la pandemia ha encendido luces de alarma sobre el procesamiento de datos y ha llevado al despliegue de medidas para proteger la privacidad de los ciudadanos y prevenir atentados contra su seguridad cibernética.

- El desarrollo de aplicaciones se ha dado no solo en el sector público (concentradas en la evolución de la pandemia y la seguridad) sino también en el sector privado. Los esfuerzos de este se dirigen en su mayoría al sector financiero y el teletrabajo, donde la amenaza a la ciberseguridad aumenta con las interacciones.

- En algunos países se están actualizando los marcos regulatorios e institucionales. Los principales cambios apuntan hacia una mayor coordinación de los esfuerzos para combatir la pandemia mediante la creación de autoridades de protección de datos y la implementación de sistemas de evaluación de la protección. 
- La emergencia sanitaria ha dado una connotación distinta a las acciones desplegadas por los gobiernos. En el cuadro 4 se muestra la forma de implementación de las estrategias de utilización de datos de países de la región. En la Argentina, el Brasil y el Ecuador, la declaración de emergencia ha permitido que los datos puedan ser extraídos por las autoridades sin consentimiento de las personas.

Cuadro 4 | América Latina (10 países): extracción de datos personales por la emergencia sanitaria, julio de 2020

\begin{tabular}{|c|c|c|c|}
\hline Pais & Declaración de emergencia & $\begin{array}{l}\text { Aplicación a } \\
\text { nivel nacional }\end{array}$ & $\begin{array}{l}\text { Se requiere consentimiento } \\
\text { de los usuarios para } \\
\text { recolectar datos }\end{array}$ \\
\hline Argentina & Decisión Administrativa núm. 431/2020 & Cuidar-COVID-19 & No \\
\hline Bolivia (Estado Plurinacional de) & Circular núm. 066/2020 & Bolivia Segura & Sí \\
\hline Brasil & Ley núm. 13.979 & Coronavírus-SUS & No \\
\hline Chile & Decreto núm. 104 & CoronApp & Sí \\
\hline Colombia & Decreto núm. 417 & CoronApp & Sí \\
\hline Ecuador & Decreto Ejecutivo núm. 1017 & Salud EC & No \\
\hline México & $\begin{array}{l}\text { Acuerdo por el que se declara como } \\
\text { emergencia sanitaria por causa de fuerza } \\
\text { mayor, a la epidemia de enfermedad } \\
\text { generada por el virus SARS-CoV2 (COVID-19) }\end{array}$ & COVID-19MX & Sí \\
\hline Paraguay & Ley núm. 6524 & Covid-19 PY & No \\
\hline Perú & Decreto de Urgencia núm. 026-2020 & PerúEnTusManos & Sí \\
\hline Uruguay & Decreto núm. 93/020 & Coronavirus UY & Sí \\
\hline
\end{tabular}

Fuente: Comisión Económica para América Latina y el Caribe (CEPAL), sobre la base de información oficial de los países.

\section{- Los datos también son un desafío para la seguridad cibernética.}

- Las normativas de ciberseguridad en la región se centran en la protección de datos, fundamentalmente para evitar robos y manipulaciones indebidas de datos, trabas en el funcionamiento de los sistemas informáticos y acciones para borrar, suprimir o bloquear el acceso a los datos.

- Las normas de ciberseguridad no han considerado la protección de la infraestructura crítica, donde las amenazas pueden perjudicar el suministro de servicios públicos (agua, energía eléctrica, transporte, entre otros).

\section{Regulación y defensa de la competencia}

- La participación de las herramientas digitales en la economía ha adquirido preponderancia en los últimos años y se acelerado de manera significativa durante la crisis del COVID-19.

- En la crisis provocada por la pandemia, la política de competencia enfrenta cambios importantes, muchos de los cuales pueden perdurar y requerir una revisión de los marcos normativos e institucionales (véase el cuadro 5).

Cuadro 5 | Principales retos regulatorios de las plataformas digitales

\begin{tabular}{llll}
\hline Ámbitos & Objetivo & Nivel & Desafíos de la digitalización \\
\hline $\begin{array}{l}\text { Protección de } \\
\text { derechos }\end{array}$ & $\begin{array}{l}\text { Proteger derechos } \\
\text { de trabajadores, } \\
\text { consumidores } \\
\text { yautores }\end{array}$ & $\begin{array}{l}\text { Internacional, nacional } \\
\text { y local }\end{array}$ & $\begin{array}{l}\text { - Asegurar la sostenibilidad de los sistemas de } \\
\text { protección social y protección de los derechos } \\
\text { Equilibrio entre la innovación y la protección } \\
\text { de los derechos }\end{array}$ \\
\hline $\begin{array}{lll}\text { Competencia } \\
\begin{array}{l}\text { Gobernanza sobre } \\
\text { los datos }\end{array}\end{array}$ & $\begin{array}{l}\text { Acceso y protección } \\
\text { de datos }\end{array}$ & $\begin{array}{l}\text { Internacional, nacional } \\
\text { y local }\end{array}$ & $\begin{array}{l}\text { Definición de mercados } \\
\text { Posibles cuellos de botella }\end{array}$ \\
\hline Fiscalidad & $\begin{array}{l}\text { Cumplimiento de la política } \\
\text { tributaria }\end{array}$ & $\begin{array}{l}\text { Internacional, nacional de reglas sobre acceso, portabilidad } \\
\text { y local }\end{array}$ & $\begin{array}{l}\text { - Acuerdos para gravar el consumo transfronterizo } \\
\text { de servicios digitales }\end{array}$ \\
\hline
\end{tabular}

Fuente: Comisión Económica para América Latina y el Caribe (CEPAL). 
- Para el mundo de la pospandemia, en el que se habrá fortalecido el rol de las tecnologías digitales, en general, y el de las plataformas, en particular, es fundamental generar los marcos normativos y regulatorios que eviten el abuso de poder de mercado derivado de la concentración e incentiven la competencia. El modelo de regulación dependerá del tipo de plataformas en cuestión: de acceso a información o contenido a través de motores de búsqueda generales; de acceso a datos personales y otro contenido privado; de acceso a bienes o servicios ofrecidos por terceros o plataformas de la llamada economía colaborativa; de acceso a la fuerza laboral o a competencias particulares según las áreas de especialización y de acceso a dinero o capital, como sitios de microfinanciación colectiva (crowdfunding), sistemas de pago o criptomonedas.

- En el gráfico 17 se muestra la tendencia del valor de mercado de las principales plataformas del mundo y de la región. Durante la pandemia, la esencialidad de los servicios digitales ha aumentado el valor de mercado de las empresas del sector digital, y en particular las plataformas. Esto contrasta con la situación de muchas empresas de la economía analógica perjudicadas por la suspensión de las actividades económicas y la interrupción de las cadenas de valor.

Gráfico 17 | América Latina y el Caribe y mundo: valor de mercado de las mayores plataformas, diciembre de 2019 a julio de 2020

(En miles de millones de dólares)

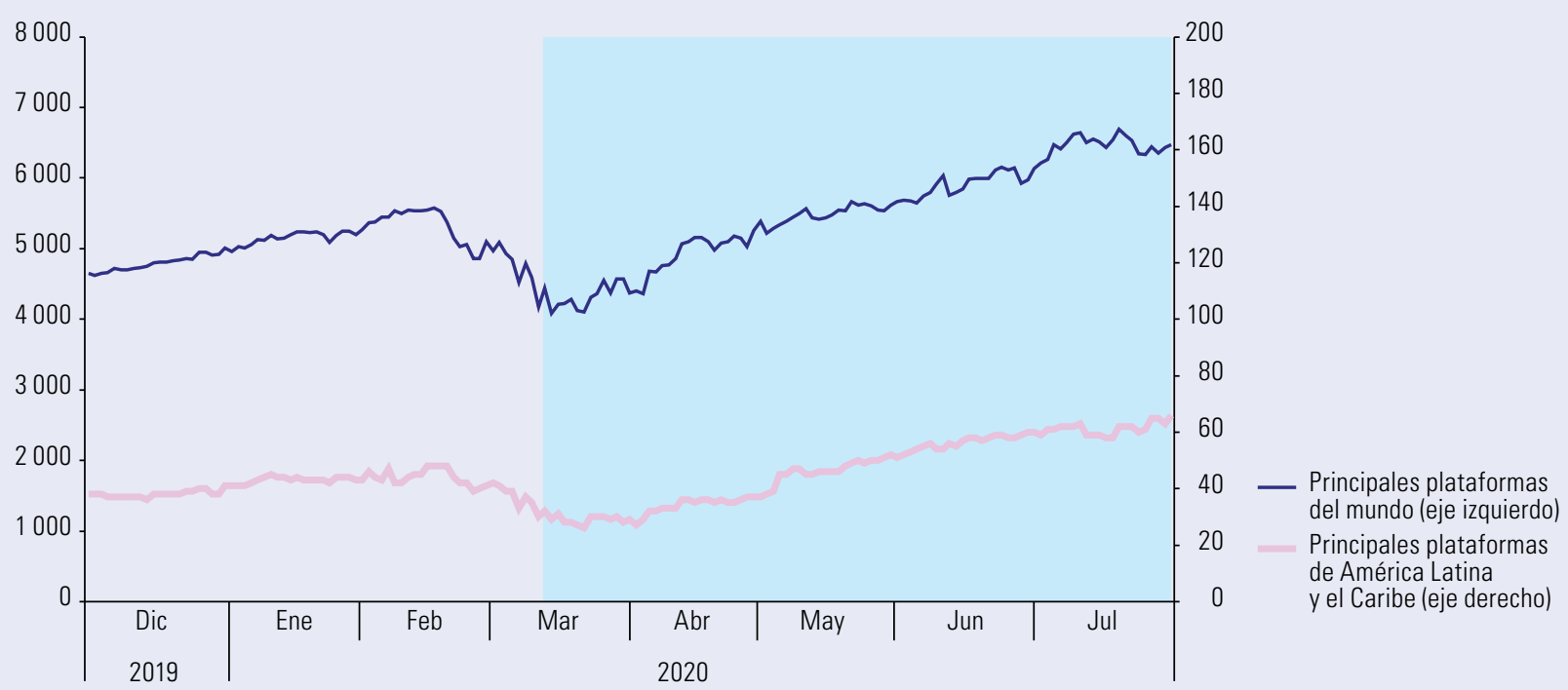

Fuente: Comisión Económica para América Latina y el Caribe (CEPAL), sobre la base de datos de Macrotrends [en línea] macrotrends.net.

Nota: Las mayores plataformas del mundo incluyen a Alphabet, Amazon, Microsoft, Apple y Facebook; las mayores de América Latina y el Caribe a PagoSeguro y MercadoLibre.

- Es necesario reducir las distorsiones a la competencia y la innovación que favorezcan a los gigantes tecnológicos, sin obstaculizar la innovación y el emprendimiento de empresas emergentes (start-ups) nacional o regional.

- La tributación de la economía digital puede representar un volumen importante de recursos fiscales. Sus modelos de negocio de la economía digital plantean problemas para los sistemas tributarios, como la ausencia de presencia física de las empresas debido a que la provisión del servicio trasciende los límites geográficos, una alta dependencia de activos intangibles difíciles de valorar, una elevada complejidad de las transacciones y dificultades para categorizar el tipo de actividad económica y los ingresos asociados.

- Ante la dificultad de aplicar impuestos directos a las empresas digitales, la mayoría de los países ha optado por aplicar impuestos indirectos como el impuesto al valor agregado (IVA) a los servicios digitales. En el mundo, 77 países han introducido este tipo de gravamen y 12 de ellos pertenecen a América Latina y el Caribe. En lo que respecta a los impuestos directos a servicios digitales, aplicables a los ingresos o ganancias de empresas no residentes que proporcionan publicidad digital, servicios o contenido a una base de usuarios locales, 22 países han adoptado medidas fiscales unilaterales en tanto se acuerden soluciones integrales de carácter internacional.

- La pandemia obliga a acelerar los esfuerzos para aumentar la contribución de la economía digital a la tributación mediante acuerdos multilaterales para evitar efectos fiscales y comerciales perjudiciales, y aumentar la participación de los países de la región en las negociaciones mundiales sobre los impuestos a la economía digital a fin de que se consideren las necesidades e intereses regionales. 
- Al mismo tiempo, el uso masivo de algoritmos de gestión para el teletrabajo, las tecnologías de la información y las comunicaciones, los grandes datos para la generación de aplicaciones de rastreo de contactos (contact tracing) para contener los contagios, la inteligencia artificial, la salud electrónica y el comercio electrónico, ha puesto de manifiesto la necesidad de establecer estándares que contemplen los marcos regulatorios antimonopolio actuales.

- En el centro de las disrupciones se encuentran las estrategias de negocio de las grandes empresas tecnológicas y plataformas (Big Tech) basadas en un mayor acceso a datos. Esto exige un debate regulatorio y de políticas, que abarque tanto la protección de datos y la privacidad como la interoperabilidad, el acceso a datos con fines de desarrollo, las políticas de datos abiertos, la propiedad intelectual y las políticas de competencia.

- La preocupación de algunos países y organizaciones por los temas de privacidad se relaciona con los mecanismos de competencia y el creciente poder de mercado de estas grandes empresas tecnológicas.

- Algunas plataformas, como Twitter, habían anunciado, ya antes de la pandemia, restricciones a los contenidos de carácter político considerados nocivos. Si bien no sería una regla general, la declaración de pandemia ha abierto la puerta a la supervisión de contenidos por parte de las empresas propietarias de las plataformas. Es preciso contar con guías o reglas claras que busquen el equilibrio entre la prohibición de contenidos nocivos y la restricción a la libertad de expresión.

- El argumento de esas empresas se basa en la responsabilidad que tienen en la difusión de información oficial relevante y veraz sobre el avance de la pandemia, y las medidas adoptadas por los gobiernos para combatirla. Sin perjuicio de reconocer la importancia del argumento, hay preocupación por la posibilidad de que estas herramientas se instalen como una nueva práctica de control y dominio de mercado por esas empresas.

\section{B. Medidas de política y soluciones digitales}

- Los Gobiernos de la región han aprobado medidas para preservar y garantizar la conectividad y los servicios de telecomunicaciones (véase el gráfico 18). Entre ellas, destacan las dirigidas a garantizar la accesibilidad, disponibilidad y asequibilidad de los servicios de telecomunicaciones, las que buscan habilitar servicios de comunicación de emergencia, gestionar el tráfico para mejorar la eficiencia de las redes y permitir el acceso gratuito a ciertas aplicaciones y sitios web, y las vinculadas a la protección del consumidor y la preservación de la calidad del servicio.

Gráfico 18 | América Latina y el Caribe (16 países)a: medidas adoptadas por los países en materia de telecomunicaciones para hacer frente a los efectos de la enfermedad por coronavirus (COVID-19), por tipo, 2020 (En número de medidas)

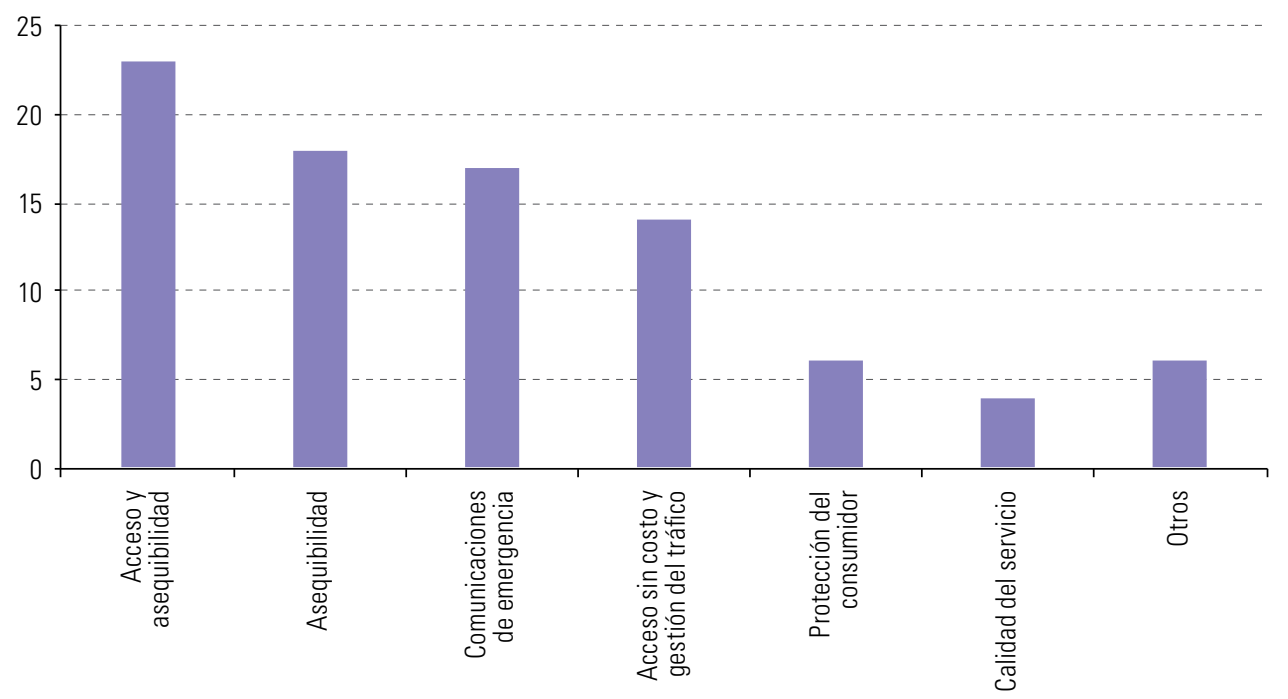

Fuente: Comisión Económica para América Latina y el Caribe (CEPAL), sobre la base de Unión Internacional de Telecomunicaciones (UIT), Plataforma de Resiliencia de la Red Mundial (\#REG4COVID), 2020 [en línea] https://reg4covid.itu.int/ y Corporación Andina de Fomento (CAF) y otros, Las oportunidades de la digitalización en América Latina frente al COVID-19, Santiago, 2020.

a Argentina, Brasil, Bolivia (Estado Plurinacional de), Chile, Colombia, Costa Rica, Cuba, Ecuador, El Salvador, Guatemala, México, Panamá, Paraguay, Perú, República Dominicana y Venezuela (República Bolivariana de). 
- Accesibilidad y banda ancha: iniciativas que facilitan el despliegue de infraestructura, como la otorgación temporal de espectro o la ampliación temporal de los topes de tráfico en los planes de Internet de banda ancha.

- Asequibilidad: los Gobiernos han instado a los operadores a prestar servicios de Internet fija y móvil de bajo costo y a no cortar el suministro de dichos servicios por falta de pago.

- Canales de comunicación de emergencia: instalación de números específicos y centros de llamadas (call centers).

- Gestión del tráfico: medidas que buscan priorizar el tráfico de datos en las áreas de salud y educación electrónica, así como permitir el acceso sin costo a ciertas aplicaciones móviles o sitios web.

- Protección del consumidor: los reguladores han optado por exigir a los operadores a que cualquier medida que deban tomar para garantizar la provisión de los servicios sea transparente. Todos los países incluidos en el análisis ${ }^{3}$ han adoptado medidas específicas para mantener la continuidad y la expansión de los servicios de telecomunicaciones.

- Por otro lado, los países han desarrollado soluciones para atender las necesidades de prevención y comunicación relacionadas con el COVID-19, así como para llevar a cabo un proceso de control y seguimiento más centrado en la pandemia y sus efectos y facilitar los tratamientos (véanse el diagrama 1 y el cuadro 6).

- En materia de prevención y comunicación, se han desarrollado canales de comunicación e información en línea; especialmente, mediante sitios web, aplicaciones y redes sociales.

- El tráfico de los portales gubernamentales ha aumentado considerablemente. Entre los nuevos servicios y funcionalidades, pueden encontrarse recursos con información general sobre la pandemia y las medidas de prevención, sistemas automáticos de comunicación (chatbots o asistentes virtuales, entre otros) y estadísticas, datos y mapas actualizados en tiempo real. Asimismo, se han elaborado plataformas sobre teletrabajo y programas de educación en línea.

Diagrama 1 | América Latina (16 países): ámbitos de aplicación de las soluciones digitales implementadas para hacer frente a la pandemia de la enfermedad por coronavirus (COVID-19), 2020

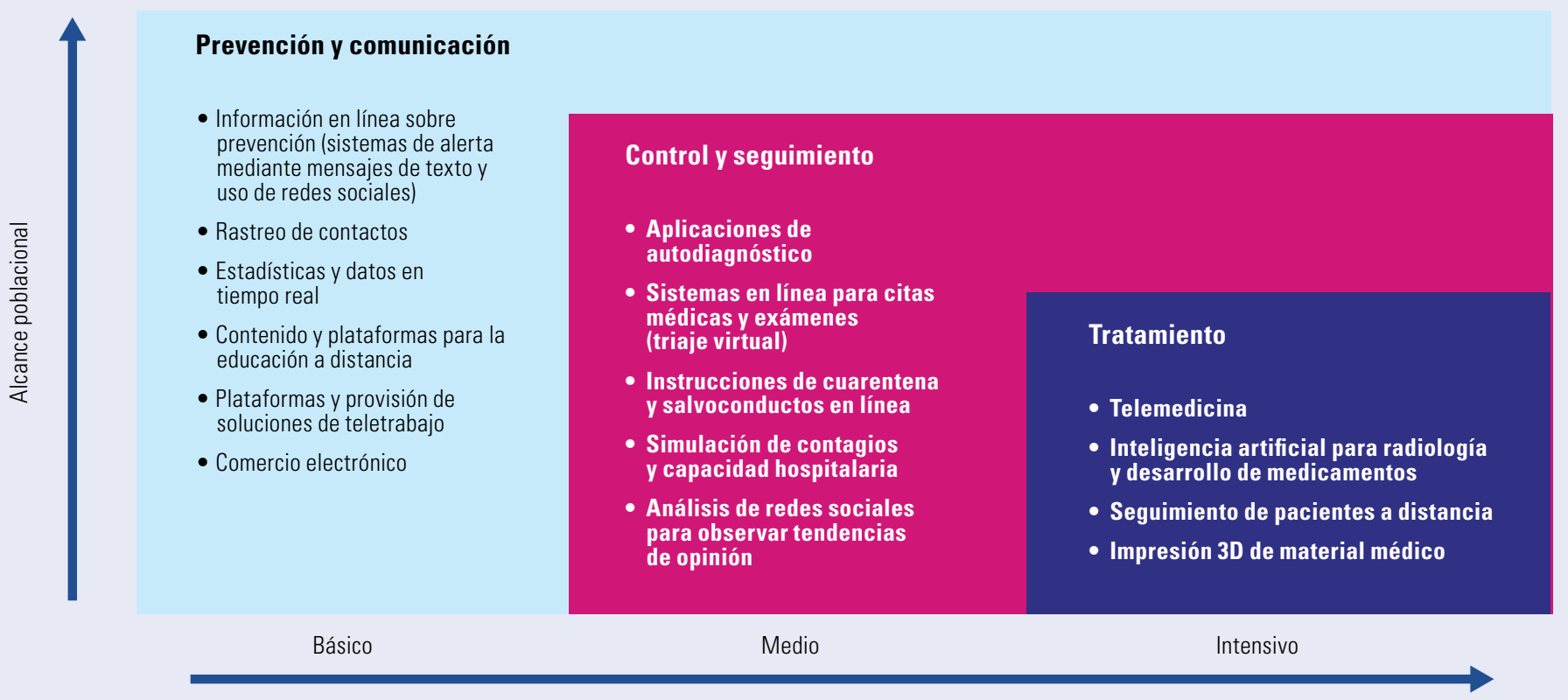

Requisitos de tecnología o ancho de banda

Fuente: Comisión Económica para América Latina y el Caribe (CEPAL).

Argentina, Brasil, Bolivia (Estado Plurinacional de), Chile, Colombia, Costa Rica, Cuba, Ecuador, El Salvador, Guatemala, México, Panamá, Paraguay, Perú, República Dominicana y Venezuela (República Bolivariana de). 
Cuadro 6 | América Latina y el Caribe (9 países): medidas adoptadas por los países vinculadas directamente a la lucha contra la enfermedad por coronavirus (COVID-19), abril de 2020

\begin{tabular}{|c|c|c|c|c|c|c|c|c|c|c|}
\hline Ámbito & Medida de política & $\begin{array}{c}\text { Bolivia (Estado } \\
\text { Plurinacional de) }\end{array}$ & México & Brasil & Ecuador & Colombia & Argentina & Uruguay & Chile & Perú \\
\hline \multirow{3}{*}{$\begin{array}{l}\text { Prevención y } \\
\text { comunicación }\end{array}$} & Rastreo de contactos & & & & & & & & & \\
\hline & $\begin{array}{l}\text { Sistemas automáticos de } \\
\text { comunicación }\end{array}$ & & & & & & & & & \\
\hline & Programas de educación en línea & & & & & & & & & \\
\hline \multirow{4}{*}{$\begin{array}{l}\text { Control y } \\
\text { seguimiento }\end{array}$} & Aplicaciones de autodiagnóstico & & & & & & & & & \\
\hline & Triaje virtual & & & & & & & & & \\
\hline & Instrucciones de cuarentena & & & & & & & & & \\
\hline & $\begin{array}{l}\text { Simulación de contagios y capacidad } \\
\text { hospitalaria }\end{array}$ & & & & & & & & & \\
\hline \multirow{4}{*}{ Tratamiento } & Inteligencia artificial para radiología & & & & & & & & & \\
\hline & $\overline{\text { Seguimiento de pacientes (chatbots) }}$ & & & & & & & & & \\
\hline & $\begin{array}{l}\text { Impresión de material médico } \\
\text { con impresoras 3D }\end{array}$ & & & & & & & & & \\
\hline & $\begin{array}{l}\text { Investigación y desarrollo (I+D) } \\
\text { para enfrentar el COVID-19 }\end{array}$ & & & & & & & & & \\
\hline
\end{tabular}

Implementada
En desarrollo
No se considera

Fuente: Comisión Económica para América Latina y el Caribe (CEPAL), sobre la base de información oficial

- En relación con el control y el seguimiento, los países han desarrollado soluciones de autodiagnóstico que se aplican mediante aplicaciones móviles y sitios web. El triaje virtual o agendamiento de citas a distancia es una solución que se ha implementado para mejorar la eficiencia de los servicios médicos.

- Las tecnologías más avanzadas, como la inteligencia artificial, son menos utilizadas. En un primer momento, la escasez de material médico fue un problema crítico. Los gobiernos y la sociedad civil respondieron mediante iniciativas relacionadas con la impresión 3D. Varios países han informado que han implementado este tipo de soluciones o las están desarrollando. Existe todavía espacio para la elaboración de políticas e instrumentos para hacer frente al COVID-19 mediante el uso de tecnologías digitales, sobre todo en el ámbito del tratamiento de los pacientes.

- Destacan medidas como la definición de las plataformas de despacho a domicilio como un servicio esencial y la autorización para que puedan operar durante las cuarentenas. Entre otras medidas que se han implementado en menor grado, se incluyen regulaciones específicas para el tratamiento de los datos personales y acciones en materia de ciberseguridad. 


\section{Hacia la reactivación}

- La crisis económica y social y las medidas de distanciamiento físico han impulsado cambios en los modelos de gestión de la producción, de demanda y de negocios que privilegian los canales en línea (véase el diagrama 2). La aceleración de la transformación digital productiva y de consumo es inevitable.

Diagrama 2 | América Latina y el Caribe: hacia la reactivación, 2020



Fuente: Comisión Económica para América Latina y el Caribe (CEPAL).

- Además, las cadenas de valor y las empresas se han visto afectadas por una menor demanda de productos. Esta situación ha impulsado cambios en los modelos de producción, que deben contar con una mayor capacidad de reacción y flexibilidad operativa. La automatización y el uso de herramientas inteligentes de producción y operación constituyen soluciones a estas necesidades.

- La crisis, que profundiza las desigualdades estructurales, obliga a priorizar el bienestar social, la resiliencia productiva y la sostenibilidad.

- El impacto de las soluciones digitales aumenta según los requisitos de las distintas etapas de la pandemia (véase el diagrama 3), el nivel de sofisticación tecnológica o el ancho de banda al que tienen acceso los países.

- Reacción. Las primeras medidas de reacción apuntaron a generar y difundir información sanitaria y aplicar medidas de distanciamiento físico. El teletrabajo y la educación en línea surgieron como primeras soluciones.

- Recuperación. En el ámbito sanitario, el rastreo de contagios permite comprender mejor la evolución de la pandemia y detectar los focos de contagio, lo que contribuye a mejorar el diseño de estrategias.

- Reactivación. El desarrollo y la adopción de tecnologías digitales en todas las áreas del sistema productivo será fundamental. 
Diagrama 3 | América Latina y el Caribe: nuevas realidades y necesidades de sofisticación tecnológica, 2020



Fuente: Comisión Económica para América Latina y el Caribe (CEPAL).

\section{La pospandemia: nueva realidad y cambio estructural}

- La nueva demanda estará basada en canales en línea y se caracterizará por un aumento de la demanda de educación en línea (cursos y contenidos en línea), salud electrónica (consultas y diagnósticos remotos), compras en línea, servicios de banca y seguros en línea y entretenimiento en línea.

- Ante este aumento de la demanda, los países y el sector privado deberán mejorar la experiencia de compra (seamless experience), los canales en línea de compra y atención al cliente, los medios de pago electrónicos, la privacidad de los datos y la seguridad digital.

- La nueva oferta estará basada en la flexibilidad, la cercanía local y la capacidad de reacción. Para ello, habrá que desarrollar nuevos productos y servicios, flexibilizar y redimensionar las capacidades, optimizar el desempeño, invertir en investigación y desarrollo (I+D) y crear o renovar capacidades.

- Este nuevo escenario hará necesario reconfigurar los siguientes elementos:

- Patrones de inversión, incluido el desarrollo de redes 5G

- Cadenas de suministro: cercanía de los proveedores (regionalización)

- Plantas: automatización de procesos y adopción de tecnologías avanzadas

- Fabricación, diagnóstico y mantenimiento remotos: modelo híbrido con empleados que realicen su trabajo de manera presencial y a distancia

- Datos: mayor uso, macrodatos e inteligencia artificial 
- La productividad y el cambio estructural seguirán siendo factores ineludibles para el desarrollo. La región debe avanzar hacia sistemas productivos más diversificados, homogéneos e integrados para aumentar la productividad y la inclusión productiva, lo que se traduciría en mayores niveles de empleo y salarios.

- La nueva realidad también generará reconfiguraciones en el ámbito gubernamental. Los gobiernos deberán incrementar sus esfuerzos en materia de gobierno electrónico, no solo en relación con la gestión sanitaria y socioeconómica de la pandemia, sino también con la digitalización de la totalidad de los servicios públicos.

\section{Cinco líneas de acción}

a) Construir una sociedad digital inclusiva

- El requisito esencial para lograr una participación efectiva en la era digital es contar con acceso a banda ancha de alta velocidad. Esto implica ampliar la cobertura de la banda ancha fija y mejorar la velocidad de conexión de la banda ancha móvil.

- Los costos asociados a la conexión de los hogares y a los dispositivos necesarios, sumados a las dificultades para financiar la infraestructura digital (por ejemplo, los cables de fibra óptica) constituyen barreras para la inclusión digital. Por ese motivo, es crucial abordar la cuestión de la asequibilidad del acceso y de los dispositivos.

- Con base en los costos de los planes de Internet de banda ancha móvil y fija, la CEPAL ha estimado el costo de conectar a todos los hogares sin conexión. Este costo incluye el pago mensual de una conexión fija y una conexión móvil en el caso de los hogares urbanos no conectados, y el pago de una conexión móvil en el de los hogares rurales (véase el gráfico 19).

- En los casos del Estado Plurinacional de Bolivia y El Salvador, el costo mensual de conectar a los hogares sin conexión es mucho mayor que en el del resto de los países de la región, debido al gran número de hogares desconectados y a su bajo nivel de ingresos. El costo de proveer una conexión a los dos quintiles de menores ingresos en ambos países es mayor que el costo que supondría lograr la conexión universal en el resto de los países.

Gráfico 19 | América Latina (11 países): costo mensual de conectar a los hogares sin conexión, 2018 o último dato disponible

(En porcentajes del PIB mensual)

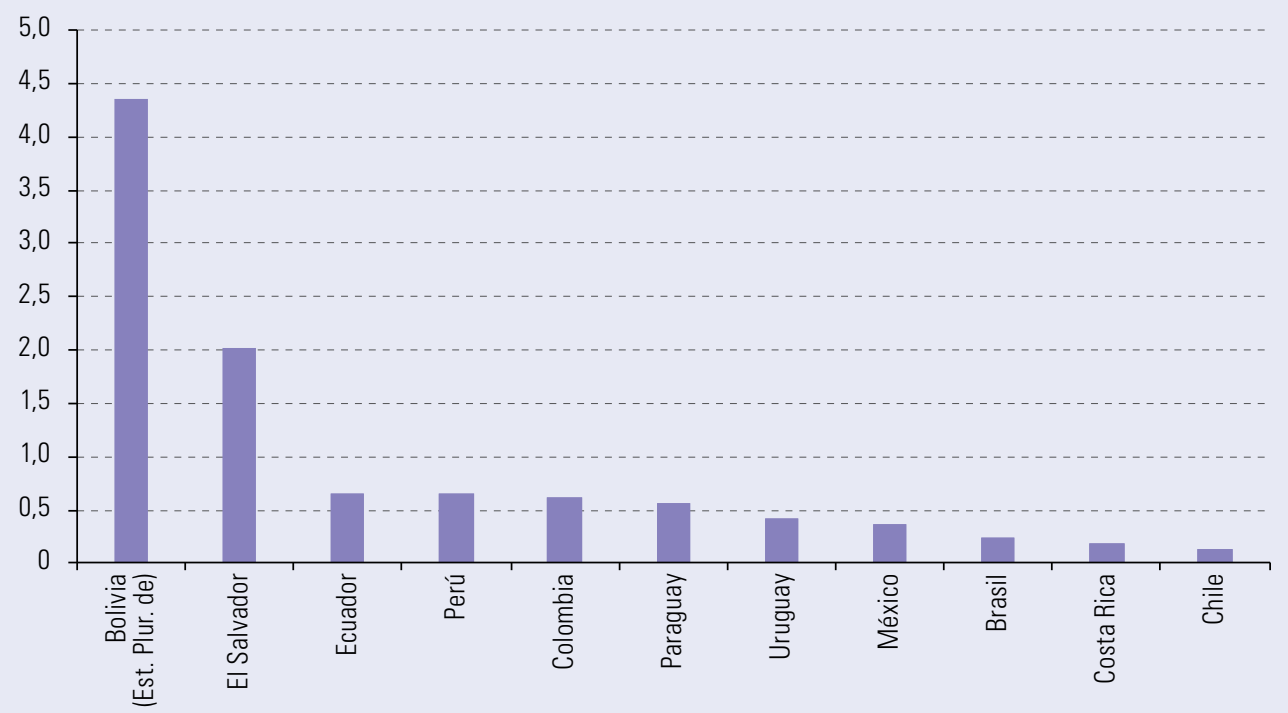

Fuente: Comisión Económica para América Latina y el Caribe (CEPAL), Observatorio Regional de Banda Ancha (ORBA), sobre la base de información del Banco de Datos de Encuestas de Hogares (BADEHOG), el Banco Mundial y sitios web de proveedores de Internet. 
- Por otro lado, la CEPAL también ha estimado el costo anual de una canasta básica integrada por una computadora portátil, un teléfono inteligente y una tableta. En el caso de muchos países de la región, es posible otorgar una canasta básica de tecnologías de la información y las comunicaciones (TIC) a los hogares que no cuentan con estos dispositivos con un costo anual inferior al $1 \%$ del PIB (véase el gráfico 20). Las medidas de apoyo a la conectividad y esta canasta de TIC son complementarias y deben operar simultáneamente para surtir efecto.

Gráfico 20 | América Latina (11 países): costo anual de una canasta básica de tecnologías de la información y las comunicaciones (TIC) para hogares sin conexión, 2019

(En porcentajes del PIB anual)



Fuente: Comisión Económica para América Latina y el Caribe (CEPAL), Observatorio Regional de Banda Ancha (ORBA), sobre la base de información del Banco de Datos de Encuestas de Hogares (BADEHOG) y el Banco Mundial.

- Para apoyar a los hogares de menores ingresos a financiar la contratación de servicios de telecomunicaciones y la canasta de dispositivos de acceso, se pueden utilizar subsidios a la demanda, para lo que es fundamental la coordinación entre el sector público y el privado.

- La CEPAL propone la implementación de un entorno de prueba (sandbox) regulatorio en el que se permita a los operadores gestionar de manera directa parte de los recursos que deben aportar los fondos de acceso universal $u$ otros fondos orientados a masificar los servicios de telecomunicaciones, para destinarlos a cubrir los costos de la provisión de servicios a los hogares de menores ingresos.

- La autorización para la gestión propia de recursos podría estar sujeta al cumplimiento de condiciones determinadas por el regulador que incentivarían la competencia entre los proveedores de servicios, lo que se traduciría en una oferta de mejores condiciones con el fin de obtener dicha autorización.

- Esta medida debe complementarse con acciones de flexibilización regulatoria, por ejemplo, en materia de neutralidad de la red, incentivando el uso de servicios de educación, salud y gobierno mediante la aplicación de tarifas cero (zero rating), que permitan el acceso a estos sin costo para el usuario.

- En relación con el acceso a los dispositivos, se propone reducir temporalmente los impuestos a las importaciones y las ventas - por ejemplo, el impuesto sobre el valor agregado (IVA) - de dispositivos definidos por el regulador, así como fomentar las alianzas público-privadas con proveedores y fabricantes para lograr condiciones más favorables en la oferta de equipos.

- Por último, para garantizar la inclusión digital, es necesario elaborar políticas a la medida de cada país y acciones que contemplen criterios socioeconómicos, geográficos, etarios $y$ de género. 


\section{b) Impulsar una transformación digital productiva}

- El escaso nivel de digitalización de las empresas de la región —sobre todo, de las micro, pequeñas y medianas empresas (mipymes) - hace necesaria la implementación de políticas públicas que permitan modificar los procesos de gestión para incorporar el uso de las tecnologías digitales en las cadenas de aprovisionamiento, procesamiento, manufactura y operaciones, así como en los canales de distribución.

- Es preciso desarrollar mecanismos de financiamiento para facilitar la creación de empresas emergentes (start-ups) y de base tecnológica.

- A fin de incentivar el fortalecimiento de los ecosistemas digitales y lograr una mayor empleabilidad, deben fomentarse la capacitación laboral y los sistemas de formación en áreas relacionadas con las tecnologías digitales.

\section{c) Promover la confianza y la seguridad digitales}

- Si bien la privacidad no puede ser un impedimento para afrontar la emergencia sanitaria, dicha emergencia no puede suponer tampoco el fin de la privacidad; es necesario alcanzar un equilibrio. Los gobiernos deben garantizar a sus ciudadanos que el acceso excepcional a los datos personales que hace falta para hacer frente a la crisis del COVID-19 conllevará una custodia adecuada de estos, y que su privacidad será resguardada.

- Además, debe definirse la responsabilidad de los funcionarios a cargo y las sanciones pertinentes por abuso o mal uso de la información personal. Es imprescindible definir marcos regulatorios que expliciten qué uso de los datos pueden hacer las empresas privadas y los gobiernos.

- La pandemia ha puesto en evidencia la necesidad de leyes — más que de decretos de necesidad y urgencia_ que determinen los mecanismos de cumplimiento de la protección de la privacidad de los datos y el establecimiento de autoridades competentes especializadas. Fortalecer la institucionalidad y los marcos normativos en el ámbito de la privacidad de los datos y la ciberseguridad es esencial.

\section{d) Fortalecer la cooperación digital regional}

- La arquitectura de la cooperación digital regional es compleja y difusa. La región carece de un marco institucional para la discusión de políticas, normas y estándares.

- La Agenda Digital para América Latina y el Caribe (eLAC 2020) surge de la Conferencia Ministerial sobre la Sociedad de la Información de América Latina y el Caribe, la cual constituye un espacio para la definición de principios y prioridades comunes, ya que convoca a 33 países de la región y a representantes del sector privado, la sociedad civil y la comunidad técnica.

- Algunos acuerdos alcanzados en el marco de la agenda eLAC 2020 son fomentar la elaboración e implementación de planes de banda ancha con metas concretas y medibles; impulsar el desarrollo y la incorporación de habilidades digitales; promover la perspectiva de inclusión en el diseño de políticas; coordinar acciones orientadas a garantizar la privacidad y la protección de datos personales, y fomentar el uso de tecnologías digitales en las empresas, con foco especial en las mipymes.

- La coyuntura requiere de una mayor articulación de la región y de los bloques subregionales para definir las prioridades temáticas y su gestión operativa.

- Para avanzar en la cooperación digital, la CEPAL propone una agenda de trabajo en las siguientes áreas: infraestructuras digitales -entre ellas, la redes de alta velocidad $5 \mathrm{G}-$ y conectividad para garantizar el acceso universal a Internet de banda ancha; protección de datos y seguridad digital; políticas de competencia y regulación, e impuestos digitales. 


\section{e) Repensar el modelo de gobernanza digital en el marco de un estado de bienestar digital}

- En el mundo posterior al COVID-19, el bienestar social no será posible sin una transformación digital. Por ello, el nuevo modelo de gobernanza digital deberá:

- Promover la igualdad, considerando procesos de transformación digital incluyentes, facilitando la interacción a través de múltiples canales con el Estado y tomando en cuenta a la población sin acceso adecuado a las tecnologías digitales, las habilidades necesarias para usarlas y las barreras socioeconómicas que restringen su aprovechamiento.

- Proteger los derechos económicos, sociales y laborales de la población, garantizando que los sistemas de protección social y sus beneficios sean provistos mediante tecnologías digitales de forma eficiente y según criterios de accesibilidad y facilidad de uso, asegurando que los procesos de selección de beneficiarios determinados por medios electrónicos sean transparentes e incluyan mecanismos para realizar aclaraciones y rectificaciones legítimas.

- Rechazar la recolección no autorizada y el uso indebido o no autorizado de datos personales, brindar altos estándares de seguridad de la información y evitar cualquier tipo de vulneración de la privacidad y la dignidad de las personas, fortalecer el acceso a la información pública y promover la lucha contra la corrupción.

- Promover un modelo de gobernanza digital desde una perspectiva estratégica productiva que genere un cambio estructural mediante la innovación y la difusión tecnológicas en el aparato productivo, la creación de nuevos modelos de negocio, la inserción en cadenas de valor globales, la generación de capacidades y habilidades digitales, y mecanismos de financiamiento que impulsen la economía digital.

Este Informe Especial es el séptimo de una serie que elabora la Comisión Económica para América Latina y el Caribe (CEPAL) sobre la evolución y los efectos de la pandemia de COVID-19 en América Latina y el Caribe. Sus análisis económicos y sociales se actualizarán a medida que surja información relevante. La Secretaria Ejecutiva de la CEPAL, Alicia Bárcena, dirigió la elaboración de este Informe, con el apoyo técnico de la Oficina del Secretario Ejecutivo Adjunto, Mario Cimoli, y de la División de Desarrollo Productivo y Empresarial de la CEPAL.

Copyright $(\subset)$ Naciones Unidas, 2020 